\title{
Discovering young stars in the Gum 31 region with infrared observations $\star, \star \star$
}

\author{
H. Ohlendorf, T. Preibisch, B. Gaczkowski, T. Ratzka, J. Ngoumou, V. Roccatagliata, and R. Grellmann
}

Universitäts-Sternwarte München, Ludwig-Maximilians-Universität, Scheinerstr. 1, 81679 München, Germany

e-mail: ohlendorf@usm.uni-muenchen.de

Received 13 August 2012 / Accepted 24 January 2013

\begin{abstract}
Context. The Gum 31 bubble, which contains the stellar cluster NGC 3324, is a poorly studied young region close to the Carina Nebula.

Aims. We are aiming to characterise the young stellar and protostellar population in and around Gum 31 and to investigate the starformation process in this region.

Methods. We identified candidate young stellar objects from Spitzer. WISE, and Herschel data. Combining these, we analysed the spectral energy distributions of the candidate young stellar objects. With density and temperature maps obtained from Herschel data and comparisons to a collect-and-collapse scenario for the region we are able to further constrain the characteristics of the region as a whole.

Results. We find 661 candidate young stellar objects from WISE data; 91 protostar candidates are detected through Herschel observations in a $1.0^{\circ} \times 1.1^{\circ}$ area. Most of these objects are found in small clusters or are well aligned with the H II bubble. We also identify the sources of Herbig-Haro jets. The infrared morphology of the region suggests that it is part of the larger Carina Nebula complex. Conclusions. The location of the candidate young stellar objects on the rim of the $\mathrm{H} \mathrm{II} \mathrm{bubble} \mathrm{is} \mathrm{suggestive} \mathrm{of} \mathrm{their} \mathrm{being} \mathrm{triggered}$ according to a collect-and-collapse scenario, which agrees well with the observed parameters of the region. Some candidate young stellar objects are found in the heads of pillars, which indicates radiative triggering of star formation. All in all, we find evidence that in the region different mechanisms of triggered star formation are at work. Correcting the number of candidate young stellar objects for contamination, we find $\sim 600$ young stellar objects in Gum 31 above our completeness limit of about $1 M_{\odot}$. Extrapolating the initial mass function down to $0.1 M_{\odot}$, we estimate a total population of $\sim 5000$ young stars for the region.
\end{abstract}

Key words. stars: formation - stars: protostars - ISM: jets and outflows - Herbig-Haro objects - ISM: clouds - ISM: bubbles

\section{Introduction}

The bubble-shaped H II region Gum 31 around the young stellar cluster NGC 3324 is located $\approx 1^{\circ}$ north-west of the Carina Nebula (NGC 3372; see Smith \& Brooks 2008 for a recent review). While numerous observations of the Carina Nebula have been performed in the past few years and provided comprehensive information about the stellar populations as well as the cloud properties (Yonekura et al. 2005; Smith \& Brooks 2007; Kramer et al. 2008; Smith et al. 2010a,b; Townsley et al. 2011; Preibisch et al. 2011a,b,c, 2012; Salatino et al. 2012), the Gum 31 region has not received much attention. Despite its interesting morphology and the publicity of HST images of its western rim (Hubble News Release STScI-2008-34), the H II region and its stellar population remain rather poorly studied until today. This seems to be related to its celestial position: the closeness to the extremely eye-catching Carina Nebula has always overshadowed NGC 3324.

\footnotetext{
* This work is based in part on data collected by Herschel, an ESA space observatory with science instruments provided by European-led Principal Investigator consortia and with important participation from NASA, and on data observed by VISTA (ESO run number 088.C0117(A)), an ESO survey telescope developed by a consortium of 18 universities in the United Kingdom, led by Queen Mary, University of London.

$\star \star$ Tables 1-3 are available in electronic form at http://www . aanda.org
}

The physical relation between NGC 3324 and the Carina Nebula complex (CNC) is still unclear. Recent distance determinations of Gum 31 and NGC 3324 yielded values of $2.3 \mathrm{kpc}$ for the cluster NGC 3324 (Catalogue of Open Cluster Data; Kharchenko et al. 2005) and $2.5 \pm 0.3 \mathrm{kpc}$ for the clouds in and around Gum 31 (Barnes et al. 2010). This implies that the NGC 3324/Gum 31 region is located at the same distance as the Carina Nebula ( $\approx 2.3 \mathrm{kpc}$; see Smith $2006 \mathrm{c}$ ).

The diameter of the Gum $31 \mathrm{H}$ II region is $\sim 15^{\prime}(10 \mathrm{pc})$. This $\mathrm{HII}$ region in turn is surrounded by an expanding H I shell that encloses it almost completely (Cappa et al. 2008).

The available information about the stellar population of NGC 3324 is restricted to the three brightest stars. The brightest star of the cluster is the multiple star HD 92206, which consists of the two O6.5V stars HD 92206 A and B and the O8.5V star HD 92206 C (Maíz-Apellániz et al. 2004). Another very luminous star in the region is the A0 supergiant HD 92207 $(=\mathrm{V} 370$ Car, $V=5.49)$. This object is a very luminous $\left(\log \left(L / L_{\odot}\right)=5.56\right)$ massive $\left(M_{\text {initial }} \approx 30 M_{\odot}\right)$ evolved star (age $\approx 7 \pm 1$ Myr; Przybilla et al. 2006) that drives a very strong stellar wind $\left(\dot{M}=1.3 \times 10^{-6} M_{\odot} \mathrm{yr}^{-1}\right.$; Kudritzki et al. 1999). The membership of HD 92207 is not entirely clear: Clariá (1977) and Carraro et al. (2001) assumed it not to be part of the cluster. However, Forte (1976, based on HD 92207's being wrapped in a nebular shell associated with Gum 31) and Baumgardt et al. (2000, from proper motions) determined it to be a member. 
Carraro et al. (2001) identified 25 candidate members of NGC 3324 with optical photometry and suggested that the cluster is very young $(\$ 2-3 \mathrm{Myr})$. With three O-type stars $(M \geq$ $18 M_{\odot}$ ), the field-star initial mass function (IMF) representation by Kroupa (2002) suggests that there should be $\approx 1500$ low-mass $\left(0.1 M_{\odot} \leq M \leq 2 M_{\odot}\right)$ stars. This implies that the vast majority of the stellar population of NGC 3324 is still unknown.

In addition to the optically visible stellar cluster in the H II region, there is a population of young stars embedded in the molecular cloud surrounding the H II region. The stars in this population are only seen in infrared images (Cappa et al. 2008) or traced by their protostellar jets (Smith et al. 2010a). Cappa et al. (2008) sampled point sources in a region of $20^{\prime}$ radius centred on NGC 3324 from the IRAS, MSX, and 2MASS point-source catalogues and identified 12 (IRAS), 9 (MSX), and 26 (2MASS) candidate young stellar objects (cYSOs) using colour-colour criteria. Owing to the limited sensitivity and angular resolution of these data, the currently known few dozen embedded infrared sources represent only the tip of the iceberg; many more embedded young stellar objects (YSOs) must be present in this area and are waiting to be discovered.

In this paper, we are aiming to characterise the protostellar and young stellar population of the stellar cluster, the surrounding H II region, and its environs. Our study is based on Spitzer, WISE, Herschel, and VISTA data, which provide much better sensitivity and angular resolution than the previously existing data sets.

We describe the data sets in Sect. 2. In Sect. 3, we describe the general morphology of the infrared clouds in and around the Gum 31 region and its implications for our work. Detections of cYSOs from the infrared data, including the identification method, and their spatial distribution are described in Sect. 4. Furthermore, we analysed point-like sources as detected with both Herschel and Spitzer and derived their spectral energy distributions (SEDs; Sect. 5). We also identified likely sources of two previously detected Herbig-Haro jets (Sect. 6). Inferences are discussed in Sect. 7.

\section{Observational data}

\subsection{Spitzer images and photometry}

\subsubsection{IRAC}

We used data taken during the Spitzer cold mission phase, in July 2008, with the InfraRed Array Camera (IRAC; Fazio et al. 2004) ("Galactic Structure and Star Formation in VelaCarina” programme; PI: Steven R. Majewski, Prog-ID: 40791), retrieved through the Spitzer Heritage Archive ${ }^{1}$. The basic calibrated data were assembled into mosaics with version 18.4 .9 of the MOsaicker and Point source EXtractor package (MOPEX; Makovoz \& Marleau 2005) provided by the Spitzer Science Centre $^{2}$ (SSC). From the complete data set recorded in the observation programme, we used only a $1.0^{\circ} \times 1.1^{\circ}$ area corresponding to the Gum 31 region and a small region to the west of it. This complements the area described by Gaczkowski et al. (2013) to complete the area surveyed with our Herschel observation programme (cf. Sect. 2.2).

With the Astronomical Point source EXtractor (APEX) module of MOPEX we then performed source detection and photometry on these IRAC mosaics. Photometry was first carried out individually for each image in the stack and was subsequently

\footnotetext{
An overview of the observations used can be found in Table 1.

2 http://irsa.ipac.caltech.edu/
}

combined internally to provide photometry data for the entire mosaic. Sets of point response functions (PRFs) available from the SSC, chosen appropriately for the time of the observations, were employed. Before the mosaics were constructed, outliers were removed using the Box Outlier Detection method within MOPEX and backgrounds between the tiles were matched using the Overlap module. Subsequently, three Spitzer astronomical observation requests (AORs) each were merged into a single mosaic.

We carried out PRF-fitting photometry separately for all four IRAC bands $(3.6 \mu \mathrm{m}, 4.5 \mu \mathrm{m}, 5.8 \mu \mathrm{m}$, and $8.0 \mu \mathrm{m})$. This photometric information was then combined into a single catalogue by a simple algorithm searching for the best match in position, taking the $4.5 \mu \mathrm{m}$ band as reference. We chose this band as our reference band because it clearly is the most sensitive one and less likely to be subject to misidentifications. These are common especially in the longest-wavelength bands where random fluctuations in nebulosities can be detected as point-like sources. In consequence, the resulting catalogue is rather conservative and excludes any object not detected in the $4.5 \mu \mathrm{m}$ band. On the other hand, it minimises contamination. The total number of point-like sources detected in at least one band in the study area is 57828 .

We noticed a slight misalignment between the Spitzer bands and therefore shifted the $4.5 \mu \mathrm{m}, 5.8 \mu \mathrm{m}$, and $8.0 \mu \mathrm{m}$ band positions throughout by $-0.17^{\prime \prime},-0.10^{\prime \prime}$, and $-0.13^{\prime \prime}$ in declination with regard to the $3.6 \mu \mathrm{m}$ band which we found to be well aligned with the Two Micron All Sky Survey (2MASS; Skrutskie et al. 2006). In the resulting catalogue, the root mean square of the spatial deviations between the IRAC and the 2MASS position is $0.22^{\prime \prime}$.

Owing to the very strong spatial inhomogeneity of the cloud emission in our maps, the sensitivity for IRAC cannot be precisely quantified by a single value. Instead, we characterise it by two typical values, the detection limit and the typical completeness limit. The former is quantified by the faintest sources in our sample. For $3.6 \mu \mathrm{m}, 4.5 \mu \mathrm{m}, 5.8 \mu \mathrm{m}$, and $8.0 \mu \mathrm{m}$ these have fluxes of $100 \mu \mathrm{Jy}, 100 \mu \mathrm{Jy}, 330 \mu \mathrm{Jy}$, and $280 \mu \mathrm{Jy}$.

To estimate the completeness limits, we constructed histograms of the measured source magnitudes (Fig. 3) and estimated the point where the rise in the source count is no longer adequately described by a power-law. Although it is not a formal measure of completeness, the turnover in source count curves can serve as a proxy to show the typical values of the completeness limit across the field. In this way we estimated completeness limits of $\approx 1.5 \mathrm{mJy}, \approx 0.7 \mathrm{mJy}, \approx 1.2 \mathrm{mJy}$, and $\approx 1.6 \mathrm{mJy}$ for $3.6 \mu \mathrm{m}, 4.5 \mu \mathrm{m}, 5.8 \mu \mathrm{m}$, and $8.0 \mu \mathrm{m}$.

Comparing these to numerical models of stellar evolution (Baraffe et al. 1998), we found that for an age of 3 Myr the photospheric emission of a $1 M_{\odot}$ star is well detectable with WISE (Sect. 2.4) in the $3.4 \mu \mathrm{m}$ and $4.6 \mu \mathrm{m}$ bands while for IRAC the sensitivity extends to $\sim 0.5 M_{\odot}$. For younger stars this boundary shifts upwards so that $1 \mathrm{Myr}$ old $0.5 M_{\odot}$ stars are still detectable with WISE while with IRAC we were able to reach $\sim 0.25 M_{\odot}$. If we compare the completeness limits to the RADMC model (Dullemond \& Dominik 2004), which provides calculations of continuum radiative transfer in axisymmetric circumstellar dust distributions around a central illuminating star, we find that disk masses as low as $0.013 M_{\odot}$ would still be detectable for a $1 M_{\odot}$ YSO.

\subsubsection{MIPS}

For the detailed analysis in Sect. 5 the IRAC data were complemented by photometry on a Multiband Imaging Photometer for 
Spitzer (MIPS; Rieke et al. 2004) $24 \mu \mathrm{m}$ map ("Spitzer Followup of HST Observations of Star Formation in H II Regions" programme; PI: Jeff Hester, Prog-ID: 20726) retrieved through the Spitzer Heritage Archive. The MIPS images were searched by eye for point-like sources coinciding with the previously identified IRAC and Herschel (Sect. 2.2) sources. Because this field is only sparsely populated with point-like sources in the $24 \mu \mathrm{m}$ band, they were easily found by eye. MIPS has a point-spread function (PSF) of 6 " full width at half maximum (FWHM) in the $24 \mu \mathrm{m}$ band, slightly degrading towards the edges of the $5.4^{\prime} \times 5.4^{\prime}$ field of view, sampled on pixels with a size of $2.55^{\prime \prime}$. According to the MIPS Instrument Handbook ${ }^{3}$, the $24 \mu \mathrm{m}$ sensitivity is highly dependent on the observed sky region; in an ideal case it is expected to be $\approx 35 \mu \mathrm{Jy}$.

Source detection and PSF photometry were then performed with StarFinder (Diolaiti et al. 2000) ${ }^{4}$. The PSF was derived as a median value from four point sources. It was then fitted to each identified point source together with a slanting plane for the background and taking into account the contribution of bright stars adjacent to the fitting region. In this way the sub-sample of MIPS point-like sources defined by IRAC and Herschel detection was analysed from the brightest $(4570 \mathrm{mJy})$ to the faintest (5 mJy) objects.

\subsection{Herschel far-infrared maps}

We analysed the Gum 31 region using data from our recent Herschel far-infrared survey of the CNC. These observations were performed in December 2010 (open time project, PI: Thomas Preibisch, Prog-ID: OT1-tpreibis-1), using the parallel fast-scan mode at $60^{\prime \prime} \mathrm{s}^{-1}$. With simultaneous five-band imaging with PACS (Poglitsch et al. 2010) at $70 \mu \mathrm{m}$ and $160 \mu \mathrm{m}$ and SPIRE (Griffin et al. 2010) at $250 \mu \mathrm{m}, 350 \mu \mathrm{m}$, and $500 \mu \mathrm{m}$, two orthogonal scan maps were obtained that cover an area of $2.3^{\circ} \times 2.3^{\circ}$. The angular resolutions of the maps are $5^{\prime \prime}, 12^{\prime \prime}$, $18^{\prime \prime}, 25^{\prime \prime}$, and $36^{\prime \prime}$ for the $70 \mu \mathrm{m}, 160 \mu \mathrm{m}, 250 \mu \mathrm{m}, 350 \mu \mathrm{m}$, and $500 \mu \mathrm{m}$ band. At a distance of $2.3 \mathrm{kpc}$ this corresponds to physical scales from 0.06 to $0.4 \mathrm{pc}$.

A full description of these observations and the subsequent data processing is given by Preibisch et al. (2012) and Gaczkowski et al. (2013). Detection and photometry of pointlike sources in Herschel bands were carried out with CUTEX (Molinari et al. 2011, also used for the Hi-GAL survey), a software package developed especially for maps with a complex background. CUTEX calculates the second-order derivatives of the signal map in four directions ( $x, y$, and their diagonals), allowing the identification of point-like sources by their steep brightness gradients. A more detailed description of the photometry process is given by Gaczkowski et al. (2013).

As any source catalogue based on maps with strong and highly spatially inhomogeneous background emission, our Herschel source lists will miss some faint sources and at the same time contain a small number of spurious detections. To improve the reliability of the source catalogue, we restricted our analysis to those sources that are detected (independently) in at least two Herschel maps and for the SED construction in Sect. 5 even to those detected in at least three bands. For this, the point-like sources detected in each individual Herschel band were matched. The matching process was described by Gaczkowski et al. (2013). In the area, we detect 91 point-like

\footnotetext{
3 http://irsa.ipac.caltech.edu/data/SPITZER/docs/mips/ mipsinstrumenthandbook

4 http://www.bo.astro.it/StarFinder/index.htm
}

sources in at least two bands and 59 in at least three bands. For each of them we checked for coincidence with IRAC-identified point-like sources. We excluded all cases where either the spatial coincidence of the Herschel and IRAC sources was not clear or where more than one IRAC source appeared as a possible counterpart. This resulted in the 17 sources identified both in Herschel and in IRAC wavelengths that are analysed in Sect. 5.

The detection limit can be approximated by the fluxes of the faintest detected sources; this is in the range of $\approx 1 \mathrm{Jy}$ to $\approx 2 \mathrm{Jy}$ in our maps. An estimate for the "typical" completeness limit (i.e. the limit above which we can expect most objects in the survey area to be detected) can be derived from the histograms of the fluxes similar to those described in Sect. 2.1.1. The corresponding limits are at $\sim 10 \mathrm{Jy}, \sim 15 \mathrm{Jy}, \sim 10 \mathrm{Jy}, \sim 10 \mathrm{Jy}$, and $\sim 6 \mathrm{Jy}$ for $70 \mu \mathrm{m}, 160 \mu \mathrm{m}, 250 \mu \mathrm{m}, 350 \mu \mathrm{m}$, and $500 \mu \mathrm{m}$.

\subsection{VISTA near-infrared images}

An $H$-band image of the area around Gum 31 was obtained with the VISTA InfraRed CAMera (VIRCAM; Dalton et al. 2006) in the night of 15 January 2012 as the first observation of our Visible and Infrared Survey Telescope for Astronomy (VISTA; Irwin et al. 2004; Emerson \& Sutherland 2010) survey of the CNC (ESO run number 088.C-0117(A)). VISTA is a 4-m class wide-field survey telescope that provides a $1.3^{\circ} \times 1.0^{\circ}$ field of view. The near-infrared camera VIRCAM consists of an array of sixteen individual $2048 \times 2048$ pixel Raytheon VIRGO infrared detectors, providing more than 67 million pixels with a nominal pixel size of $0.339^{\prime \prime}$ on the sky and sensitive in a wavelength range of $0.85-2.4 \mu \mathrm{m}$. Because the sixteen chips are noncontiguous they produce a set of sixteen non-contiguous images called a "pawprint". For a contiguous sky coverage six pawprints, offset in $x$ - and $y$-direction, are combined. The resulting "tile" covers an area of $1.5^{\circ} \times 1.2^{\circ}$ on the sky.

For each of the six pawprints, 27 exposures were obtained at five jitter positions with an integration time of $2 \mathrm{~s}$ each. Because the seeing conditions (with an average FWHM of $\approx 1.7^{\prime \prime}$ ) did not meet the pre-specified quality criteria, the observations were terminated after this observing block was completed. The observations in the other filters and for the rest of our Carina Nebula mosaic positions were successfully completed a few months later and were processed by the VISTA data flow system at the Cambridge Astronomy Survey Unit. However, at the time of writing the photometric calibration of these data was still in progress. Therefore, we used only the image data for a (preliminary) scientific analysis here, but no photometric values.

A preliminary photometric calibration showed that objects as faint as $H \approx 18.5$ are clearly detectable in our VISTA image. This is about four magnitudes deeper than the nominal 2MASS Survey completeness limit for crowded locations near the galactic plane of $H \approx 14.5$ (Skrutskie et al. 2006).

\subsection{WISE}

We used catalogue data from the Wide-field Infrared Survey Explorer (WISE; Wright et al. 2010) All-Sky Data Release (Cutri \& al. 2012). These data were taken during the WISE cold mission phase from January to August 2010. We used the standard aperture-corrected magnitudes obtained with apertures of $8.25^{\prime \prime}$ and corrections of $0.222 \mathrm{mag}, 0.280 \mathrm{mag}$, and $0.665 \mathrm{mag}$ for $3.4 \mu \mathrm{m}, 4.6 \mu \mathrm{m}$, and $12 \mu \mathrm{m}$ (Cutri et al. 2012). The WISE observations have an angular resolution of $6.1^{\prime \prime}, 6.4^{\prime \prime}, 6.5^{\prime \prime}$, and 12.0" for $3.4 \mu \mathrm{m}, 4.6 \mu \mathrm{m}, 12 \mu \mathrm{m}$, and $22 \mu \mathrm{m}$ (Wright et al. 2010). 


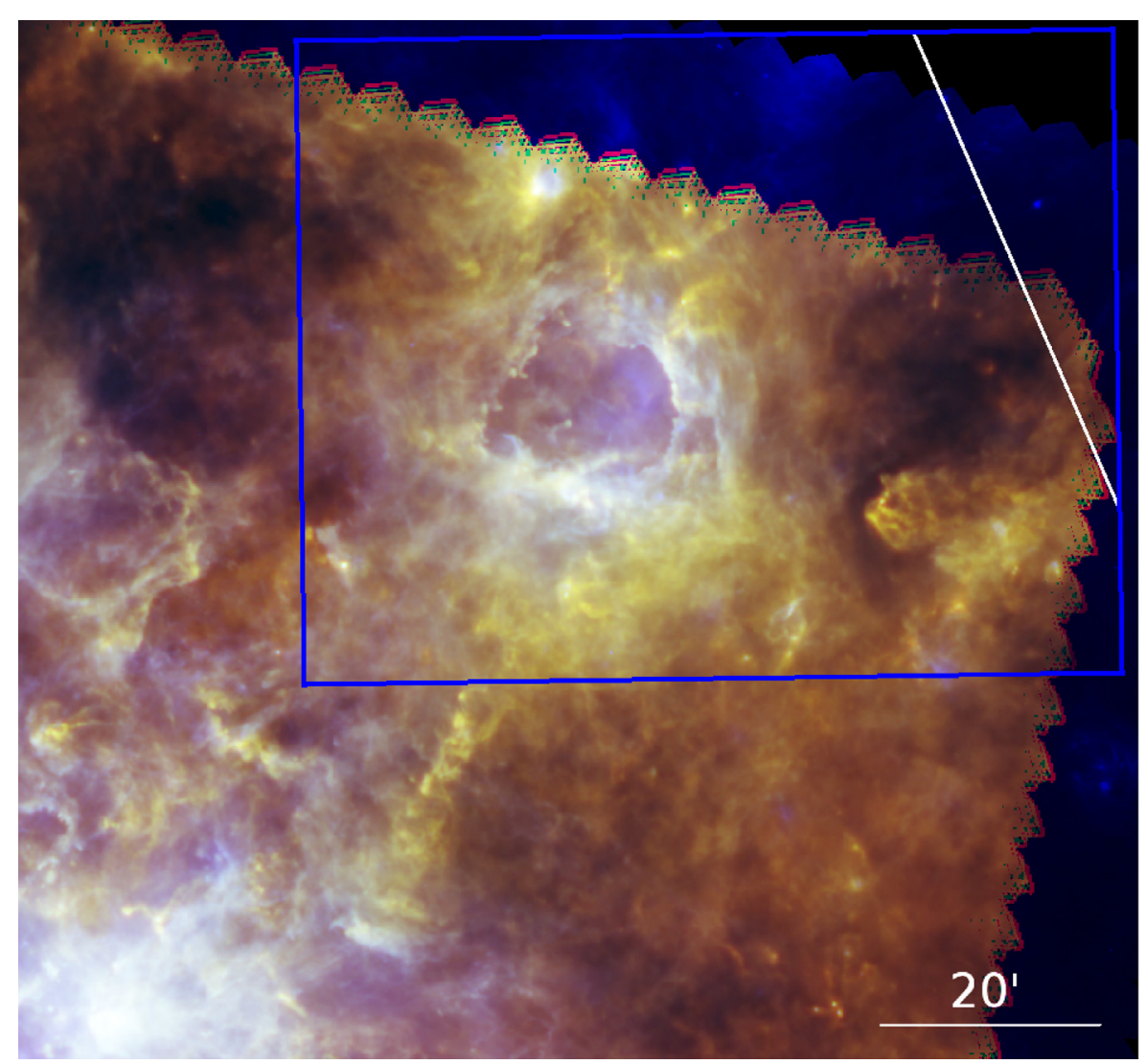

Fig. 1. Gum 31 region and its connection to the central Carina Nebula, seen in a Herschel RGB image, with the PACS $70 \mu \mathrm{m}$ image in blue, SPIRE $250 \mu \mathrm{m}$ in green, and SPIRE $500 \mu \mathrm{m}$ in red. The blue box marks the $1.1^{\circ} \times 1.0^{\circ}$ area used for analysis here. The diagonal white line marks the border to the left of which we obtained IRAC photometric data.
In our survey area the catalogue contains 20739 point sources with a detection in at least one band. Source confusion in the catalogue should not constitute a problem in the analysis here because we required a matching radius of $0.6^{\prime \prime}$ to match WISE to IRAC detections, the same as we used for the IRAC inter-band matching. On this scale, we were able to distinguish clearly between nearest and second-nearest neighbours.

Similarly to what we described in Sect. 2.1.1, we estimated the detection limits to be $90 \mu \mathrm{Jy}, 70 \mu \mathrm{Jy}$, and $700 \mu \mathrm{Jy}$ for $3.4 \mu \mathrm{m}$, $4.6 \mu \mathrm{m}$, and $12 \mu \mathrm{m}$ (for the analysis described in Sect. 4.2 we did not use the $22 \mu \mathrm{m}$ band). Analogously, we estimated completeness limits of $4 \mathrm{mJy}, 3 \mathrm{mJy}$, and $24 \mathrm{mJy}$.

\section{Morphology}

Figure 1 shows the far-infrared morphology of the Gum 31 bubble and its environs; the cavity of the H II region is clearly delineated. Figure 2 contrasts optical and infrared images of the Gum 31 bubble. Near the centre of the bubble a cluster of stars is just discernible in the IRAC image. This is NGC 3324. In the MIPS $24 \mu \mathrm{m}$ and PACS $70 \mu \mathrm{m}$ images the warm dust surrounding the stars of NGC 3324 forms an arc-like structure that follows the shape of the inner bubble wall.

Several pillar-like structures extend from the edge of the bubble into its inner part, especially from its southern rim. Notably, some but not all optical pillars coincide with those seen in the infrared. The photodissociation regions that very sharply delineate the edge of the bubble are clearly observable in the IRAC $5.8 \mu \mathrm{m}$ image, which shows their fluorescence under the influence of UV radiation. They are also visible in the MIPS $24 \mu \mathrm{m}$ image, which shows the emission from the dust grains within.

Figure $4 \mathrm{a}$ shows a colour-temperature map for the larger Gum 31 region, extending downwards into the central CNC. It was derived from the ratio of the PACS $70 \mu \mathrm{m}$ and $160 \mu \mathrm{m}$ emission as detailed by Preibisch et al. (2012). It shows that the temperatures for the HII region are comparatively high $(\approx 30-40 \mathrm{~K})$, while those of the surrounding clouds are considerably lower down to $20 \mathrm{~K}$. The warm dust in NGC 3324 is clearly seen as an outstandingly blue $(\approx 40 \mathrm{~K})$ patch inside the bubble.

The G286.21+0.17 cluster (cf. Sect. 4.4.4) also stands out. It is about $8 \mathrm{~K}$ warmer than its immediate surroundings. It is also much denser, as can be seen from Fig. 4b, which shows the hydrogen column density derived from the colour temperatures as described by Preibisch et al. (2012). We see that the column density inside the bubble is relatively low compared to its surroundings at a few $10^{20} \mathrm{~cm}^{-2}$ and rises steeply by more than one order of magnitude at the ionisation front. Apart from G286.21+0.17, we see some more dense clumps scattered within the bubble rim and beyond it. A notable feature is the cluster G286.38-0.26 (cf. Sect. 4.4.5), which has a column density of around $3 \times 10^{22} \mathrm{~cm}^{-2}$. In sharp contrast to the unusually warm clump G286.21+0.17, in Fig. 4a G286.38-0.26 is seen to be cooler than its surroundings, down to $\approx 20 \mathrm{~K}$. The integrated cloud mass derived by Preibisch et al. is $186700 M_{\odot}$. These authors derived this value by integrating over the same columndensity map that we show here, but for a region around Gum 31 slightly differently defined than ours.

In the longer IRAC-wavelength emission and especially in the Herschel image, a "bridge" of filamentary structure can be seen to extend from Gum 31 downwards in the direction of the central Carina Nebula, forming a connection. The column density map (Fig. 4b), too, shows that the clouds surrounding Gum 31 are connected to the clouds in the more central parts of the Carina Nebula. Forte (1976) remarks that in the deep optical plates of Lyngå \& Hansson (1972) a filamentary structure can be seen that connects the H II region $\mathrm{H}-31$ ( $\widehat{=}$ Gum 31; Hoffleit 1953) to NGC 3372, the central Carina Nebula. 

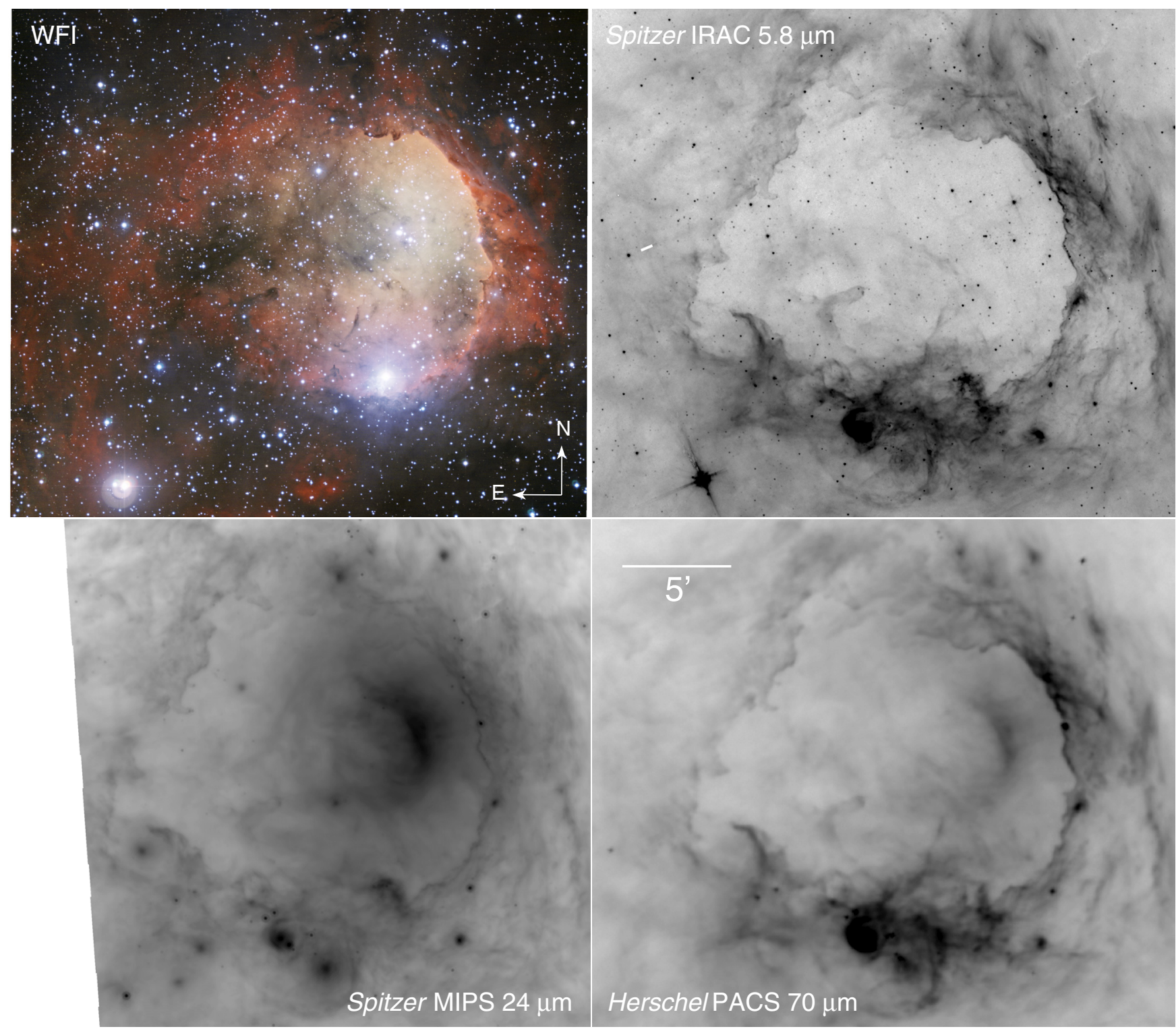

Fig. 2. Overview of the Gum 31 region in the optical, mid-infrared, and far-infrared. The ESO Wide Field Imager (WFI) optical image [ESO image release eso1207] shows the $V$ band in blue, $R$ band in yellow, O III $501 \mathrm{~nm}$ emission in green, and $\mathrm{H} \alpha$ in red.

According to Yonekura et al. (2005), the radial velocities of their $\mathrm{C}^{18} \mathrm{O}$ clumps Nos. 2-6, which surround the Gum 31 bubble, range from $V_{\mathrm{LSR}}=-20.0 \mathrm{~km} \mathrm{~s}^{-1}$ to $V_{\mathrm{LSR}}=-24.1 \mathrm{~km} \mathrm{~s}^{-1}$. The $\mathrm{C}^{18} \mathrm{O}$ clumps in the central and northern part of the Carina Nebula (Nos. 8-12) have radial velocities in the $V_{\mathrm{LSR}}=$ $-25.8 \ldots-19.9 \mathrm{~km} \mathrm{~s}^{-1}$ range. This good agreement of the radial velocities suggests that the clouds around Gum 31 and the Carina Nebula are connected and part of the same complex. As was argued by Preibisch et al. (2012) and in the introduction, we therefore assumed a distance of $2.3 \mathrm{kpc}$ towards Gum 31, the same as to the CNC. This number also agrees well with recent independent distance determinations (see Sect. 1).

\section{Young stellar objects in the Gum 31 region}

\subsection{Identification of $Y S O$ candidates}

The selection of cYSOs in the following sections is based on the detection of infrared excesses. It follows, therefore, that only YSOs exhibiting excess infrared emission can be detected with these methods (cf. also Sect. 4.3). Infrared excess emission is indicative of circumstellar material. This means that only YSOs of
Class 0 to Class II are the subject of this analysis, while Class III objects must remain undetected ${ }^{5}$.

\subsubsection{Selection by Spitzer colours}

With IRAC data only and following the criteria established for a survey of the star-forming region Pismis 24 (Fang et al. 2012; Allen et al. 2004), we were able to identify 304 infrared excess sources from plotting the [3.6]-[4.5] colour against the [5.8]-[8.0] colour for those 6739 point-like sources detected in all four bands. To identify an object as a cYSO, Fang et al. (2012) demand (Eq. (2) follows Allen et al. 2004):

$$
\begin{aligned}
& {[3.6]-[4.5] \geq 0 \quad \text { and } \quad[5.8]-[8.0] \geq 0.4} \\
& {[3.6]-[4.5] \geq 0.67-([5.8]-[8.0]) \times 0.67}
\end{aligned}
$$

The resulting colour-colour diagram is shown in Fig. 5.

5 YSOs are commonly classified according to the slope of their SEDs (Lada 1987), from Class 0 protostars in the main collapse phase through Class I YSOs shrouded in envelopes and accreting infalling circumstellar matter and Class II YSOs, T Tauri stars with disks, to Class III objects with little or no circumstellar matter. 

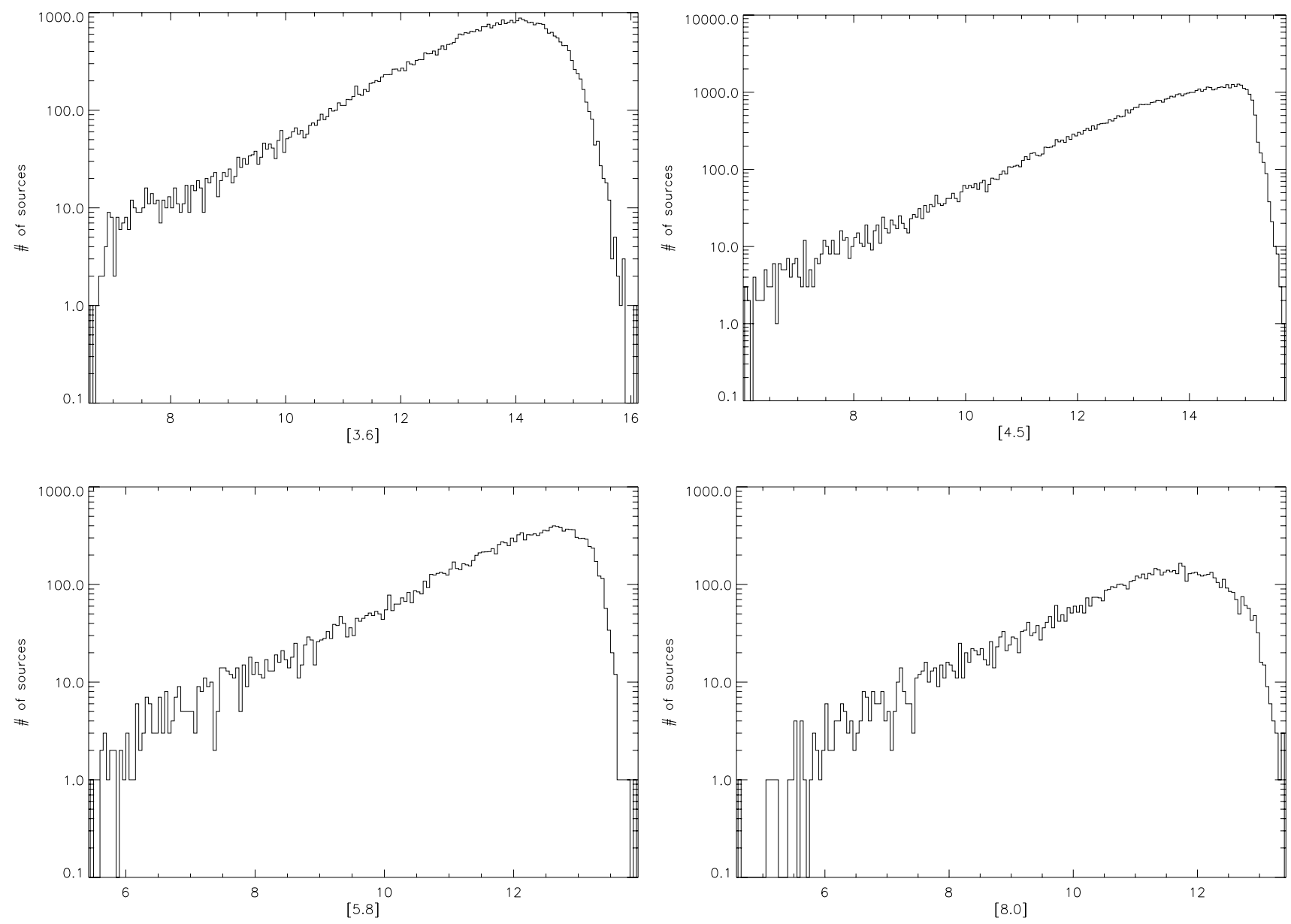

Fig. 3. Histograms of the measured source magnitudes in our Spitzer IRAC catalogue.

If we also take into account the 2MASS magnitudes, we can sample a much larger portion of sources by applying the excess criteria of Winston et al. (2007, using Spitzer extinction as derived by Flaherty et al. 2007). These yield another estimate of the YSOs in the region from the $J-H$ vs. $H-$ [4.5] colour-colour diagram. In this way, $2577 \mathrm{cYSOs}$ are identified (from a total sample of 38459 with detections in the three bands).

Within this sample, there are certainly contaminants. Asymptotic-giant-branch stars unfortunately exhibit very similar colours to YSOs and so do background star-forming galaxies. In a spectroscopic survey of objects discovered by the Spitzer Legacy Program "From Molecular Cores to Planet-Forming Disks" ("c2d") in the Serpens molecular cloud Oliveira et al. (2009) found a contamination of $25 \%$ within their subsample of the brightest objects $\left(F_{8.0 \mu \mathrm{m}}>3 \mathrm{mJy}\right)$. They attributed this high proportion to the closeness of the Serpens cloud to the Galactic plane - which is also true for Gum $31\left(b_{\text {Gum } 31} \approx\right.$ $0.2^{\circ}$ ). We can also estimate the contamination based on the criteria of Winston et al. (2007) for the selection of contaminants from the [5.8]-[8.0] vs. [4.5]-[5.8] and [4.5]-[8.0] vs. [3.6]-[5.8] diagrams. This yields an estimated contamination in the IRAC-selected sample of $\lesssim 10 \%$.

It is noticeable that the distribution of these cYSOs throughout Gum 31 and outside it is almost uniform. This is suspicious. We therefore conclude that the contamination from fore- and background sources is high and do not use this sample in our analysis.

\subsection{Selection by WISE colours}

We used WISE catalogue data to search for infrared excess sources. Koenig et al. (2012) developed a set of criteria in their study of massive star-forming regions to identify likely YSOs from WISE four-band photometry. We applied these criteria accordingly.

Using the [3.4]-[4.6] and [4.6]-[12] colours, partly in combination with the [3.4] and [4.6] magnitudes, probable background objects were removed from the sample before the YSOs were selected. This includes galaxies (very red in [4.6]-[12]), broad-line active galactic nuclei (of similar colours as YSOs, but distinctly fainter) and resolved polyaromatic hydrocarbon emission regions (redder than the majority of YSOs). From this cleaned sample the infrared excess sources are then selected by demanding (Eqs. (3) and (4): Koenig et al. 2012)

$[3.4]-[4.6]-\sigma_{1}>0.25$ and [4.6]-[12] $-\sigma_{2}>1.0$,

where $\sigma$ is the quadratically added uncertainty of the respective magnitudes. Class I sources are a subsample of this defined by

$[3.4]-[4.6]>1.0$ and $[4.6]-[12]>2.0$

(the rest are Class II objects). From this analysis we excluded any data point in the catalogue that did not have a signal-to-noise ratio of 5 or better. Figure 6 shows the [3.4]-[4.6] vs. [4.6]-[12] colour-colour diagram, constructed after probable contaminators were removed. The total sample before this removal consisted of 10128 sources. After removing probable contaminators, 6669 sources remained. These are shown in Fig. 6. Class I 
H. Ohlendorf et al.: Discovering young stars in the Gum 31 region with infrared observations
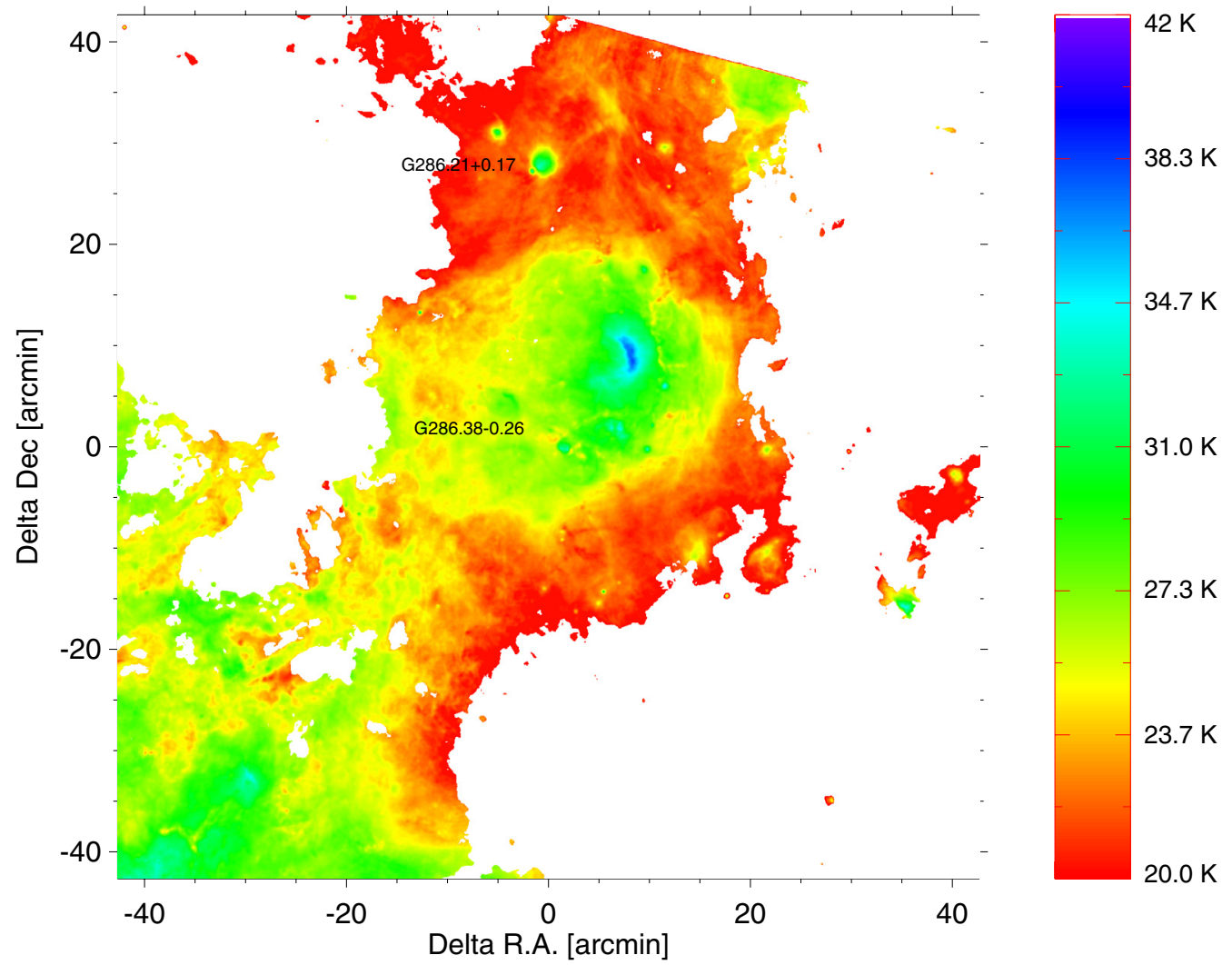

(a) Colour-temperature map.

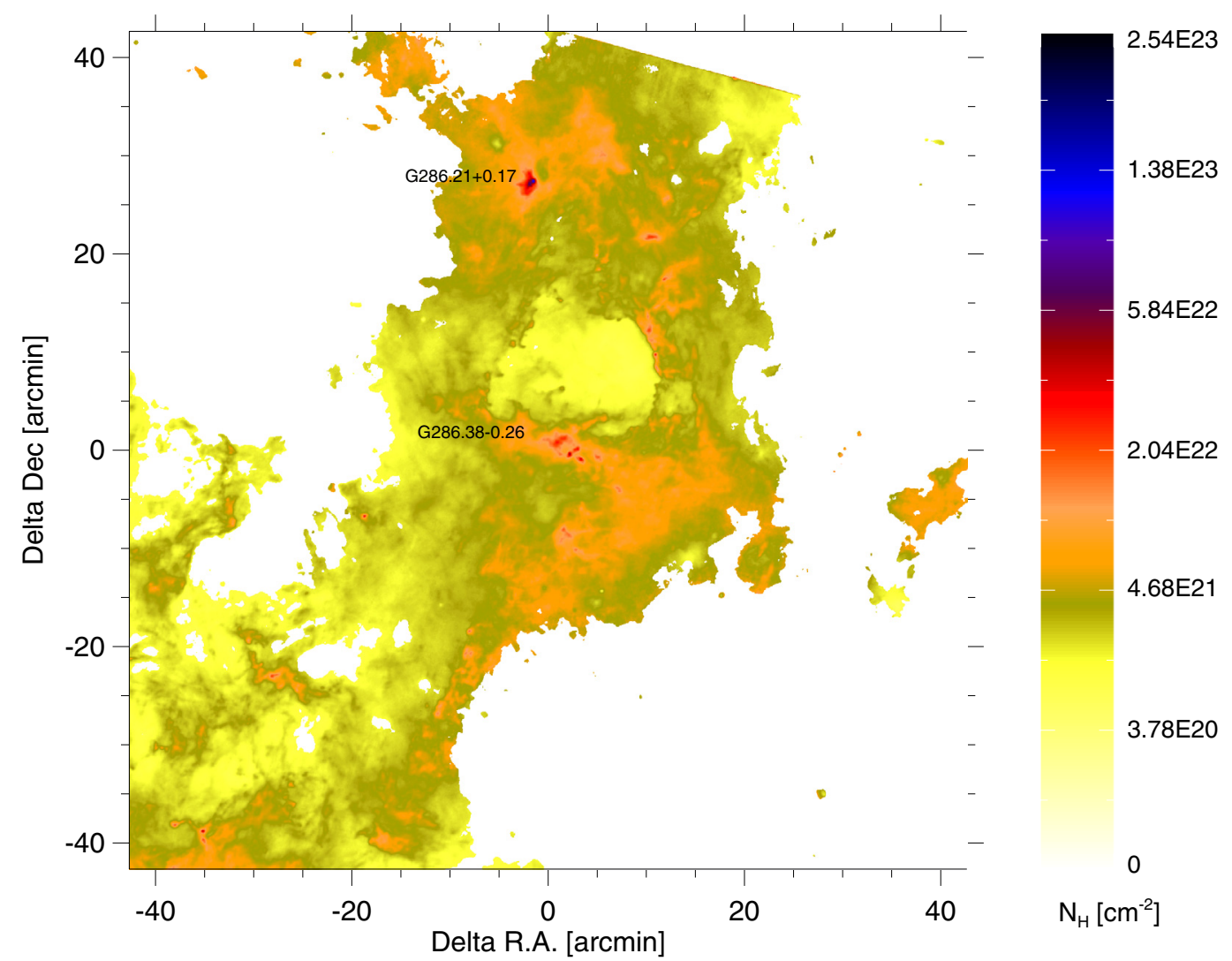

(b) Hydrogen column-density map.

Fig. 4. Maps derived from Herschel data. They show the cloud structure in and around the Gum 31 nebula and the connection to the central parts of the Carina Nebula (just outside the lower left edge of the maps). 


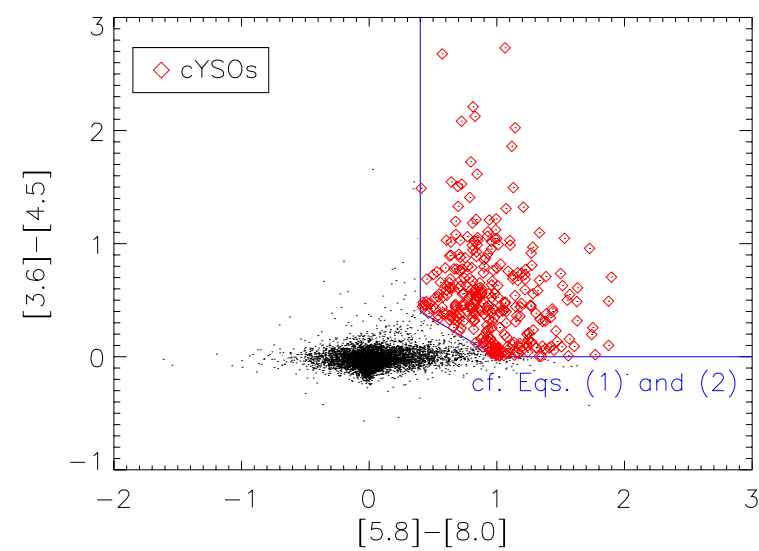

Fig. 5. Colour-colour diagram using all Spitzer IRAC bands. The criteria adopted for YSO identification follow Fang et al. (2012) and Allen et al. (2004).

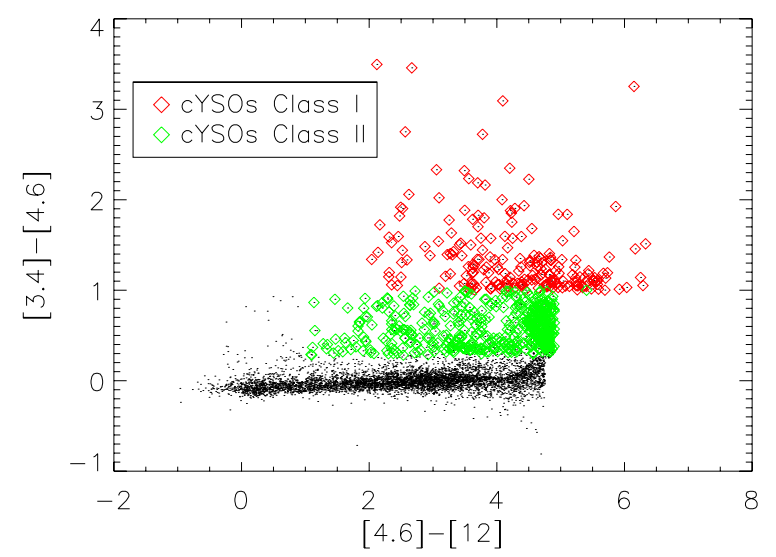

Fig. 6. Colour-colour diagram using three of the four WISE bands. The criteria adopted for YSO identification follow Koenig et al. (2012). Candidate YSOs are marked in red for Class I sources and in green for Class II.

sources are marked in red, Class II in green. The same colourcoding is used in Fig. 8, which shows the spatial distribution of the cYSOs. This analysis yields 661 cYSOs, of which 207 are Class I and 454 are Class II.

The distribution of WISE-selected cYSOs follows the cloud structure as expected. Koenig et al. (2012) estimate the remaining contamination of the cYSO population selected with their criteria by galaxies to be $\sim 10 \mathrm{deg}^{-2}$, which in our $1.2 \mathrm{deg}$ field leads to an estimate of $\sim 12$. However, in a region projected onto the Galactic disk such as the Carina Nebula we expect a high level of contamination mainly from background and foreground stars. To estimate the total contamination we performed the same analysis as described above for two circular areas of $30^{\prime}$ radius well outside the Carina Nebula (i.e. regions that we expect to be relatively free of YSOs), one centred around $\alpha_{2000}=10: 51: 52.5$, $\delta_{2000}=-59: 14: 30$, the other around $\alpha_{2000}=10: 25: 10.7, \delta_{2000}=$ $-58: 52: 00$. They were chosen to have a similar proximity to the Galactic plane as Gum 31 ( $b=0.15$ and $b=-1.18$; Gum 31: $b=-0.17)$. Furthermore, we carefully selected fields that were as free of $\mathrm{CO}$ and $\mathrm{H} \alpha$ emission as possible and appeared as empty as possible in the IRAS $100 \mu$ m images.

In these control fields we find a mean cYSO density of $97 \mathrm{deg}^{-2}$; with the cYSOs spread homogeneously. In the Gum 31 region we determine a cYSO density of $550 \mathrm{deg}^{-2}$. This leads us to expect a contamination of around $18 \%(\sim 120 \mathrm{cYSOs})$ for the Gum 31 cYSO sample. For the IRAC data we were not able to conduct a similar comparison because we do not have photometry data for the full study area around Gum 31 and none for regions outside the central nebula.

We therefore deem the WISE-selected sample to be more reliable and base our conclusion in the following sections predominantly on this sample, although we occasionally describe a classification with IRAC. In general, though, these should be treated as less reliable.

\subsection{Identification of protostars from Herschel data}

With the methods described in Sect. 2.2 that are presented in more detail by Gaczkowski et al. (2013), we obtained a pointsource catalogue for the Herschel data. Although we consider only point-like Herschel sources in the following, it is important to keep in mind that the relatively large PSF corresponds to quite large physical scales at the $2.3 \mathrm{kpc}$ distance of Gum 31 . In the PACS $70 \mu \mathrm{m}$ map, all objects with an angular [spatial] extent of up to $\approx 5^{\prime \prime}[0.056 \mathrm{pc}]$ are compact enough to appear point-like. For the SPIRE $250 \mu \mathrm{m}$ map, this is true for sources of up to $\approx 18^{\prime \prime}[0.20 \mathrm{pc}]$. This immediately shows that (pre-stellar) cloud cores, which have typical radii of $\lesssim 0.1 \mathrm{pc}$, cannot be (well) resolved in the Herschel maps and may appear as compact and point-like sources. This implies that YSOs in all their evolutionary stages can, in principle, appear as point-like sources in our Herschel maps. However, the possibility of detecting an object in a specific stage strongly depends on its properties: As described by Gaczkowski et al. (2013), many pre-stellar cores ( $\left.\gtrsim 1-2 M_{\odot}\right)$ and embedded protostars $\left(\gtrsim 1 M_{\odot}\right)$ will be easily detectable, while most of the more evolved pre-main sequence stars with disks are likely to remain undetected. In this last case the detection limit depends on the disk mass; as shown by Gaczkowski et al., a $1 M_{\odot}$ YSO is still detectable if it has a disk mass of $\gtrsim 0.5 M_{\odot}$, this drops to $\gtrsim 0.1 M_{\odot}$ for a $3 M_{\odot}$ YSO and $\gtrsim 0.01 M_{\odot}$ for a $6 M_{\odot}$ YSO.

This means that while we find mainly Class II YSOs and some Class I YSOs with IRAC, with Herschel the emphasis is on Class 0 protostars, with some Class I stars. This also implies that the overlap between both is relatively small (cf. Sect. 5). Ragan et al. (2012) presented radiative transfer models of starless cores and protostellar cores and investigated the detectability of these classes of objects. These models showed that the SEDs of starless (i.e. pre-stellar) cores typically peak around $200-300 \mu \mathrm{m}$ and drop very steeply towards shorter wavelengths. Their model fluxes at $70 \mu \mathrm{m}$ (scaled to the distance of the CNC) are several orders of magnitude below our detection limits. Protostellar cores, on the other hand, have much stronger fluxes at PACS wavelengths. Guided by these results, we can consider a detection at $70 \mu \mathrm{m}$ as an indication for the protostellar nature of the source, whereas Herschel sources without $70 \mu \mathrm{m}$ detection would be pre-stellar cores. In Fig. 7 these two classes are indicated separately.

Gaczkowski et al. (2013) argued that a sample of Herschel point-like objects in the Carina Nebula is very unlikely to be contaminated by evolved stars or extragalactic objects. The same reasoning applies to Gum 31. The photometric data for sources that fall within the Gum 31 area can be found in the paper of Gaczkowski et al. (2013).

\subsection{Spatial distribution of the cYSOs}

It is interesting that both Class I and Class II sources are predominantly found within the interior of the Gum 31 bubble or along 


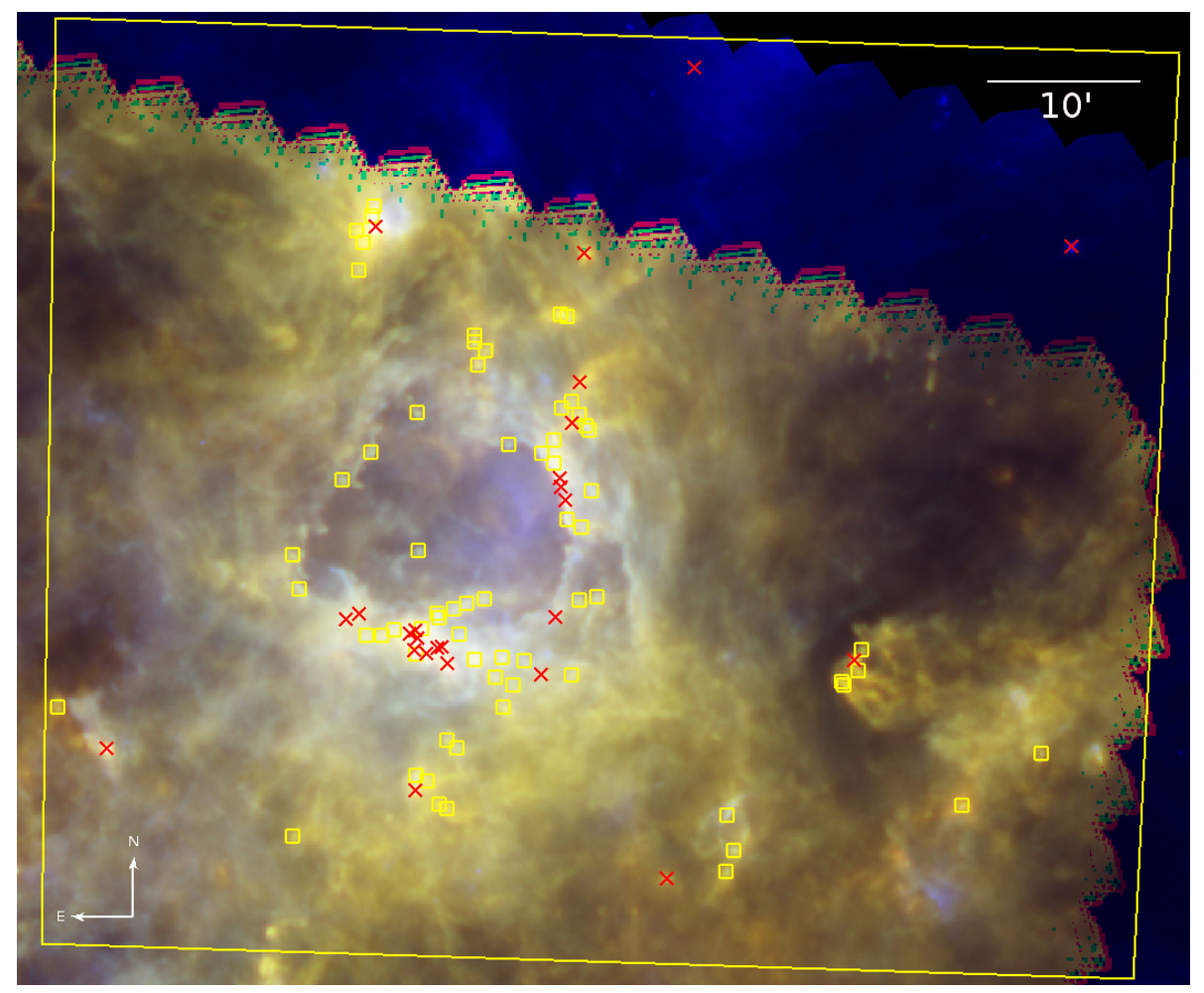

Fig. 7. Herschel RGB image (red: $500 \mu \mathrm{m}$, green: $250 \mu \mathrm{m}$, blue: $70 \mu \mathrm{m}$ ) with positions of all Herschel point-like sources detected in at least two bands overlaid. Red crosses show the position of protostellar, yellow boxes those of prestellar cores. The large yellow rectangle marks the borders of the analysed region. its rim. They tend to occur in clusters and in their distribution are often correlated with the Yonekura et al. (2005) molecular cloud clumps (see below). When we refer below to a cYSO with a number preceded by "[CNA2008]", this source is found in the catalogue of cYSOs of Cappa et al. (2008).

\subsubsection{NGC 3324}

The cluster NGC 3324 is prominent in the Spitzer images, containing $\approx 200$ point-like sources. Within the cluster itself, we identified only a single cYSO with WISE, J103706.9583710 at the western edge of the cluster (Class II). From the [3.6]-[4.5] vs. [5.8]-[8.0] diagram (IRAC) we identified six cYSOs coincident with the cluster NGC 3324 that are distributed about $40^{\prime \prime}$ towards the north-east of the cluster centre. The very small fraction of stars with detectable infrared excess $(\approx 0.5 \%)$ supports previous age estimates of $\gtrsim 3 \mathrm{Myr}$ for this cluster.

\subsection{2. cYSOs in the rim of the bubble}

Numerous cYSOs are found lined up along the ionisation front of the Gum 31 bubble to the west of NGC 3324. Fourteen cYSOs are found right behind the edge of the ionisation front. Among them are J103653.9-583719, J103653.3-583754, and J103652.4-583809, three Class I candidates that are found along the ridge traced in IRAC and Herschel images at the very edge of the bubble, neighbouring NGC 3324. Two of them, J103653.9583719 and J103652.4-583809, will be discussed in Sect. 6 as possible sources of Herbig-Haro jets.

Behind this first row of Class I candidates, there is a second row of five Class II candidates, all lined up about 19" behind the ionisation front. Farther northwards along the rim there are four more Class II candidates, one similarly behind the front and three right along it.

In Fig. 8 it is evident that a major part of the cYSOs is located along the bubble edges, similar to what is observed in comparable bubbles associated with H II regions (e.g.
Dewangan et al. 2012). Their distribution follows its circular shape and their number sharply decreases outside the cloud structure which appears magenta in the image. This is suggestive of triggered star-formation in a collect-and-collapse scenario as described by Whitworth et al. (1994).

Following analytical models for this scenario, a stellar-wind bubble produced by three massive stars with spectral types O6.5, O6.5, and O9.5 can reach a radius of approximately 9-11 pc in $1.5-2.0 \mathrm{Myr}$ assuming initial cloud densities in a range of $500-1000 \mathrm{~cm}^{-3}$. The radius at which fragmentation would occur would then vary between $7 \mathrm{pc}$ and $11 \mathrm{pc}$. To estimate the mechanical luminosities emitted by the three most massive stars in NGC 3324, we used values from Smith (2006a; and the erratum Smith 2006b) for stars with the same spectral types (luminosity class V) observed in the Carina Nebula. The $\sim 15^{\prime}$ diameter of the Gum $31 \mathrm{H} \mathrm{II}$ region corresponds to $10 \mathrm{pc}$ at a distance of $2.3 \mathrm{kpc}$, which agrees very well with the values derived from the model. That we also find numerous embedded cYSOs in and near the rim of the bubble is consistent with star-formation according to the collect-and-collapse model.

\subsection{3. cYSOs in pillars}

In the southern and eastern part of the bubble edge we found a number of short pillars extending into the bubble interior. They can be seen in the optical and infrared in Fig. 2; a close-up of the infrared pillars is shown in Fig. 9. Within four of them we found cYSOs in their very tips, reminiscent of what is observed in the central Carina Nebula (e.g. the South Pillars region). One of them, J103806.6-584002, coincides with a Herschel pointlike source (cf. Sect. 5.1) and is therefore most probably a protostar. This suggests that radiative triggering is at work, very similar to the processes seen in the South Pillars (Smith et al. 2010b; Gaczkowski et al. 2013). 


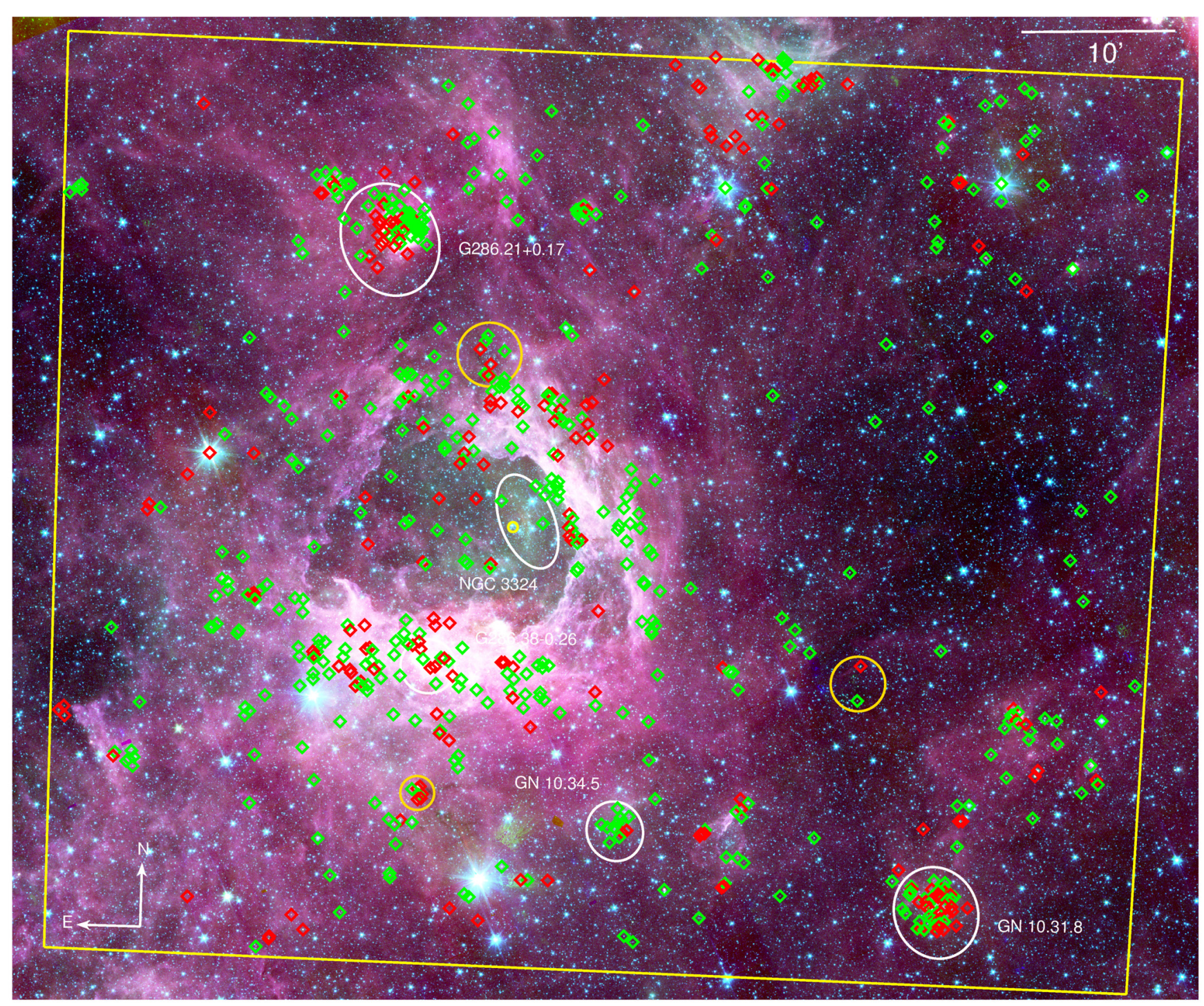

Fig. 8. Spitzer IRAC RGB image (red: $8.0 \mu \mathrm{m}$, green: $4.5 \mu \mathrm{m}$, blue: $3.6 \mu \mathrm{m}$ ) with positions of WISE-identified cYSOs overlaid. Class I sources are marked by red diamonds, Class II in green. The white ellipses represent the clusters discussed in Sect. 4.4, with the yellow circle marking the multiple star HD 92206 that contains the O stars in NGC 3324. The orange circles mark the cYSO clusters discussed in Sect. 4.4.6. The yellow rectangle marks the borders of the analysed region.

\subsubsection{G286.21+0.17}

The cloud clump G286.21+0.17 ([DBS2003]127, BYF73) is located about $12^{\prime}$ north of the rim of the Gum 31 bubble. The structure of this clump was recently studied in several molecular lines by Barnes et al. (2010). They determined a diameter of $\sim 0.9 \mathrm{pc}$, a luminosity of $\sim 2-3 \times 10^{4} L_{\odot}$, and estimated a clump mass of about $20000 M_{\odot}$ (a value that is about forty times as high as the previous mass estimate based on millimetre data by Faúndez et al. 2004). Their derived mass infall rate of $\sim 3.4 \times 10^{-2} M_{\odot} \mathrm{yr}^{-1}$ would be the highest mass infall rate seen to date, if confirmed. These properties make this cloud a particularly interesting site of possible massive-star formation.

In our Herschel maps, this clump appears as a very bright and prominent compact feature. In Fig. 11, we compare its morphology in the Herschel far-infrared bands to a near-infrared image from our VISTA data and a Spitzer mid-infrared image. As already discussed by Barnes et al. (2010), a young stellar cluster, surrounded by diffuse nebulosity, is located immediately north-west of the clump. This cluster appears very prominent in Fig. 8 as well and contains $\sim 45$ cYSOs. In the centre of the clump itself, the Spitzer images show two bright point sources with an angular separation of 7.6" (Fig. 11). They can be identified with the $10 \mu \mathrm{m}$ point sources J103832.08-581908.9 and J103832.71-581914.8 that were detected as counterparts of the MSX/RMS massive cYSO G286.2086+00.1694 described by Mottram et al. (2007), for which a bolometric luminosity of $7750 L_{\odot}$ was determined.

The VISTA $H$-band image shows very faint diffuse nebulosities at the location of these two mid-infrared sources (see the close-up in Fig. 10). This is consistent with the idea that these two objects represent deeply embedded YSOs in the protostellar evolutionary phase. With WISE the two sources are not resolved but run into one that we classify as a Class I candidate.

The peak of the Herschel PACS $70 \mu \mathrm{m}$ emission is centred on the mid-infrared source J103832.0-581908. A two-dimensional 
H. Ohlendorf et al.: Discovering young stars in the Gum 31 region with infrared observations
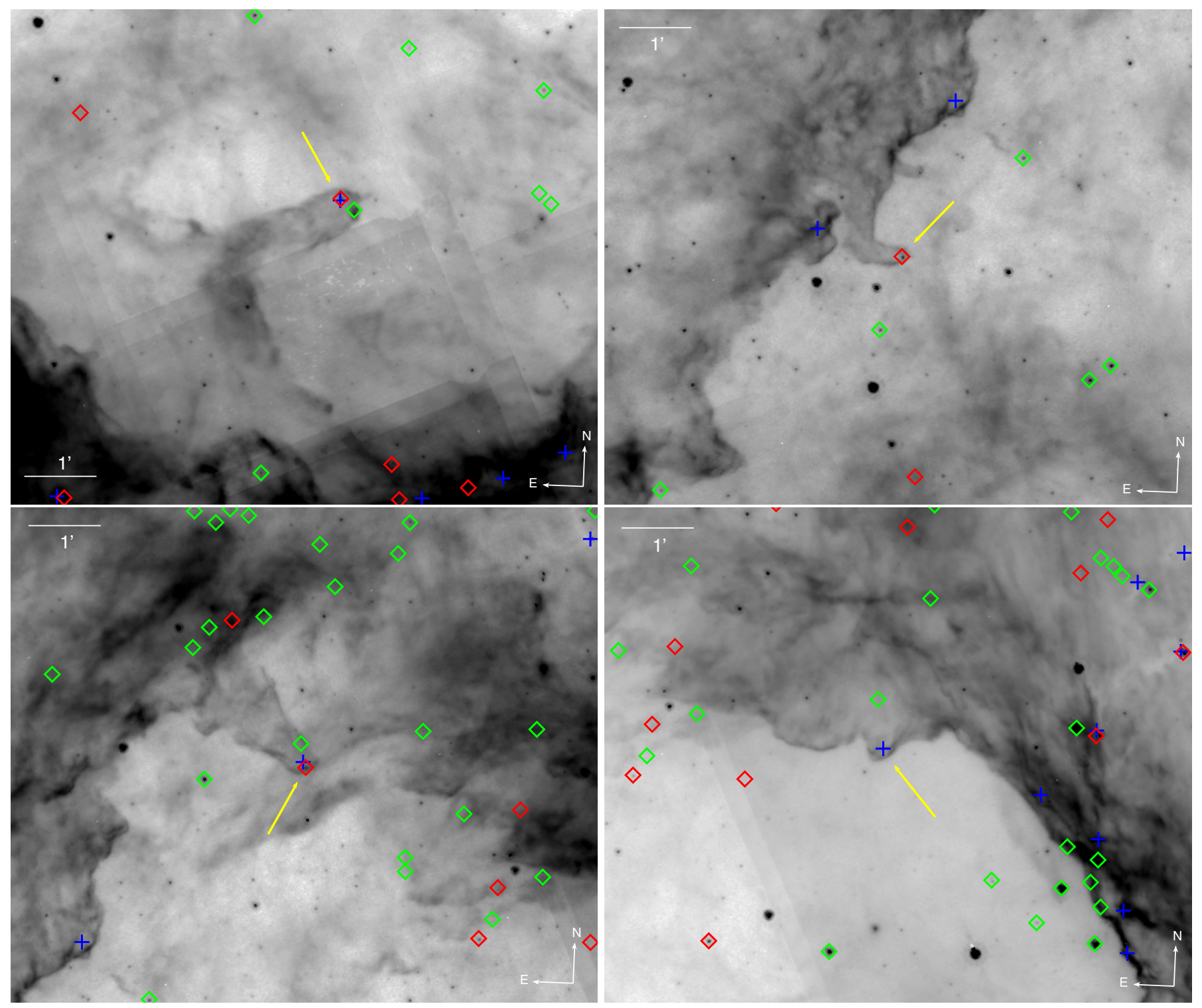

Fig. 9. In a Spitzer IRAC $8.0 \mu \mathrm{m}$ image, the yellow arrows mark the positions of four remarkable cYSOs found in the very tips of pillars. Positions of WISE-identified cYSOs are overlaid as in Fig. 8: Class I sources are marked by red diamonds, Class II sources in green. Blue crosses mark Herschel point-like sources detected in at least two bands.

Gaussian fit to the $70 \mu \mathrm{m}$ emission yields a FWHM size of $13.6^{\prime \prime} \times 11.3^{\prime \prime}$, which is clearly larger than the FWHM size of $10^{\prime \prime} \times 10^{\prime \prime}$ measured for several isolated point-like sources in the same map. With an angular distance of 7.6", the emission of the two mid-infrared sources cannot be resolved in the Herschel maps, but the measured direction of the elongation towards J103832.71-581914.8 suggests that both contribute to the observed far-infrared emission.

A second cYSO is found in the south-east of the clump, at the projection of the line connecting J103832.08-581908.9 and J103832.71-581914.8. It probably corresponds to the IRACidentified source at $\alpha_{2000}=10: 38: 33.6, \delta_{2000}=-58: 19: 22$. There is a third identified cYSO, situated at the very edge of the clump, at $\alpha_{2000}=10: 38: 35.0, \delta_{2000}=-58: 18: 44$. These three are the only cYSOs identified in the clump with WISE, while in the immediately adjoining cluster of stars visible in the IRAC images a large number of cYSOs is found. There are $\sim 45$ overall, with slightly more Class II sources than Class I sources. If there is any trend in their spatial distribution, Class I are found slightly more often to the south-east, while Class II sources tend to be located towards the north-west.

Our colour-temperature map (Fig. 4a), constructed from the Herschel $70 \mu \mathrm{m}$ and $160 \mu \mathrm{m}$ maps, shows that the cloud temperatures range from $\lesssim 20 \mathrm{~K}$ at the edge of the clump to $\sim 25-30 \mathrm{~K}$ in the clump centre, and up to $33 \mathrm{~K}$ in the nebulosity surrounding the stellar cluster north-west of the clump.

In our Herschel column-density map (Fig. 4b), the level $N_{\mathrm{H}}=2 \times 10^{22} \mathrm{~cm}^{-2}$ traces the shape of the clump. This agrees very well with the morphology as seen in the $1.2 \mathrm{~mm}$ map shown by Faúndez et al. (2004). The peak value of the column density is found to be $1.4 \times 10^{23} \mathrm{~cm}^{-2}$ and corresponds to a visual extinction of $A_{V} \approx 70 \mathrm{mag}$. From our column density map we determined the mass of the clump by integrating over a $200^{\prime \prime} \times 200^{\prime \prime}$ $(2.23 \mathrm{pc} \times 2.23 \mathrm{pc})$ box around the clump and subtracting the local background level. This yields a clump mass of $2105 M_{\odot}$. This value is nearly five times as high as the $470 M_{\odot}$ derived by Faúndez et al. (2004), but a factor of ten lower than the $20000 M_{\odot}$ estimated by Barnes et al. (2010). This seems to 


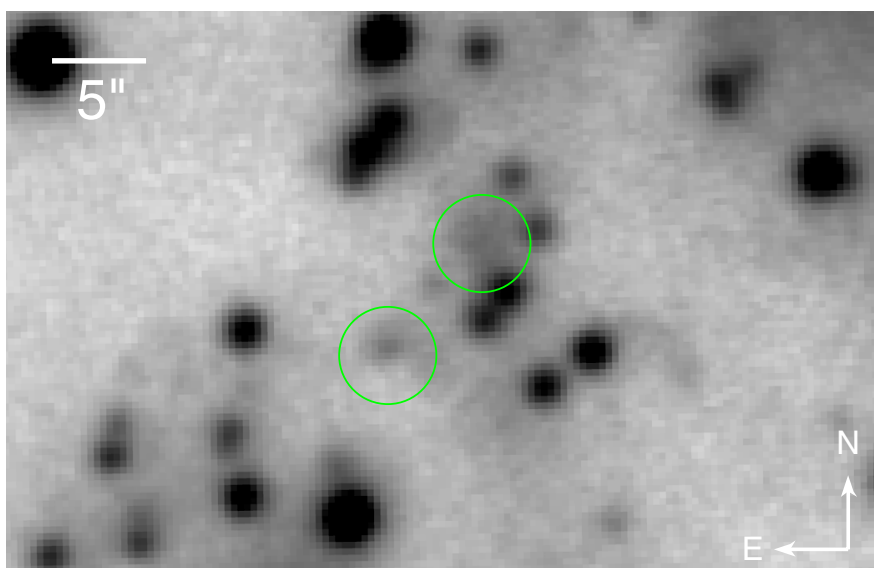

Fig. 10. Close-up of the VISTA $H$-band image (Fig. 11) around the two bright IRAC sources (green circles) within the cluster G286.21+0.17.

suggest that the mass infall rate estimated by Barnes et al. (2010) is also too high, and this clump is not as extreme as suspected.

Its far-infrared fluxes as derived from our Herschel data using elliptical apertures for photometry with GAIA ${ }^{6}$ are $1661 \mathrm{Jy}$, $2261 \mathrm{Jy}, 1293 \mathrm{Jy}, 653 \mathrm{Jy}$, and $297 \mathrm{Jy}$ for $70 \mu \mathrm{m}, 160 \mu \mathrm{m}, 250 \mu \mathrm{m}$, $350 \mu \mathrm{m}$, and $500 \mu \mathrm{m}$, which results in an integrated far-infrared luminosity (70 $\mu \mathrm{m}$ to $1.3 \mathrm{~mm}$ ) of $L_{\text {int }} \approx 9000 L_{\odot}$.

This is clearly one of the most luminous clumps in the CNC. Its mass is quite high, but probably not as high as previously suggested. It may form stars with $\lesssim 10 M_{\odot}$, but is probably not massive enough to the form high-mass stars with $M_{*} \gtrsim 20 M_{\odot}$.

\subsubsection{G286.38-0.26}

The Spitzer IRAC images show a prominent dense cluster of several dozen stars at the southern edge of the Gum 31 bubble, which is surrounded by bright diffuse nebulous emission (Fig. 12). This cluster is listed as [DBS2003] 128 by Dutra et al. (2003). It is spatially coincident with the extended $(r=2 \mathrm{pc})$ $\mathrm{C}^{18} \mathrm{O}$ clump ${ }^{7}$ No. 6 (Yonekura et al. 2005).

The nebulosity around the stellar cluster displays a remarkable arc-like shape at the eastern edge. Projecting it into a full circle, it would have a diameter of about $42^{\prime \prime}$ in IRAC images and 62" in Herschel images. The centre of this circle would be around $\alpha_{2000}=10: 38: 03, \delta_{2000}=-58: 46: 19$. The star J10380461-5846233, cYSO [CNA2008] 21, is found close $\left(\sim 11^{\prime \prime}\right)$ to this central position of the arc. In the Spitzer bands, the cYSO shows strongly increasing brightness with wavelength. In the MIPS $24 \mu \mathrm{m}$ image, it is the brightest point-source in the cluster. It was detected as a mid-infrared source by MSX and is listed as G286.3773-00.2563 in the MSX6C catalogue. From the WISE data we classify it as a Class II cYSO. The star is not detected in any of our Herschel far-infrared images. Using USNO-B optical catalogue data, 2MASS, IRAC, MIPS, and WISE photometry and Herschel upper limits we employed the online SED fitter by Robitaille et al. (2007) to construct an SED and thus estimate the (proto-) stellar parameters. The stellar mass is estimated to be $\approx 5.8 M_{\odot}$ for the best-fit model, the

\footnotetext{
6 http://astro.dur.ac.uk/ pdraper/gaia/gaia.html

We note that Yonekura et al. (2005) denoted these structures as "cores"; however, according to the definition that cores are very compact clouds (with typical sizes of $\sim 0.1$ pc or less), out of which individual stellar systems form, these clouds are better characterised as clumps (i.e. relatively large dense clouds linked to the formation of small stellar clusters).
}

luminosity $\approx 238 L_{\odot}$. Within the arc three other WISE Class I cYSOs are seen, J103805.8-584542, J103758.4-584648, and J103800.7-584654.

The optically brightest star in the cluster is HD 303094, for which a spectral type A2 is given by Nesterov et al. (1995). It is located about 17" south of J10380461-5846233 and the centre of the arc. According to the Pickles \& Depagne (2010) survey of all-sky spectrally matched Tycho-2 stars, it may be a foreground star (distance: $886 \mathrm{pc}$ ).

Strong far-infrared emission from the region of this cluster was detected with IRAS (point source IRAS 10361-5830). Our Herschel maps resolved this far-infrared emission into ten point-like sources in the area of the clump. The only Herschel source with a near-infrared counterpart is J103801.4-584641. The IRAC image at this point is dominated by strong nebular emission. There is extended emission in a confined region a few arcseconds east of the Herschel-identified point-like source, but since it is not well-resolved and the identification with the Herschel-identified source is ambiguous, we do not include it in the sample studied in Sect. 5. The two Herschel-identified point-like sources north-east of the arc-like nebula that are also detected as bright sources in the MIPS maps are J103810.2584527 and J103807.2-584511; both have no clear near-infrared counterparts. A similar case is J103754.0-584614, which has a very faint nebulous near-infrared counterpart in the VISTA image. All three are also detected as point-like sources in our IRAC images. They are included in Table 2 (online material), but we obtained the model parameters listed in Table 3 (online material) only for J103807.2-584511 because for the others the quality of the SED fit was not sufficient. Additionally, all three sources described are classified as Class I sources from WISE data.

In the south-western part of this extended $\mathrm{C}^{18} \mathrm{O}$ clump, Yonekura et al. (2005) detected a compact $(r=0.27 \mathrm{pc}) \mathrm{H}^{13} \mathrm{CO}^{+}$ clump (their clump No. 2); with a central density of $n\left(\mathrm{H}_{2}\right)=$ $6.8 \times 10^{4} \mathrm{~cm}^{-3}$ this is the densest of all the clumps they detected in their survey of the CNC. The Herschel-identified pointlike source J103750.8-584718 coincides with this clump. With IRAC, there are several small point-like sources seen to be coincident with it and an identification is therefore impossible. An IRAC-detected source slightly to the south-west of it, $\mathrm{J} 103749.3-584722$, is identified as a Class I WISE cYSO.

This area also contains the A0 supergiant HD 92207. This star has a strong near-infrared excess, was detected as a $12 \mu \mathrm{m}$ source with IRAS (IRAS 10355-5828), and is seen as a pointlike source surrounded by nebulosity in the MIPS $24 \mu \mathrm{m}$ image. Neither the star nor the surrounding nebulosity can be seen in the Herschel images.

\subsubsection{Other structure}

Towards the south-west of the Gum 31 shell, two reflection nebulae are found, GN 10.34.5 and GN 10.31.8 (both are marked with white ellipses in Fig. 8). They are very clearly delineated by cYSOs and coincide with two of the most conspicuous clusters in our field of view. GN 10.34.5 is visible in the Spitzer $\mathrm{red} / \mathrm{green} / \mathrm{blue}$ (RGB) image (especially the $4.5 \mu \mathrm{m}$ band) and seen in projection with about ten cYSOs. GN 10.31.8, on the other hand, is both larger in angular extent and coincident with four times as many cYSOs. It, too, is very conspicuous in the $4.5 \mu \mathrm{m}$ band.

There is also a smaller cluster of about twenty IRAC pointlike sources centred at $\alpha_{2000}=10: 38: 03, \delta_{2000}=-58: 55: 09$ within the Gum 31 shell (orange circle in Fig. 8). Immediately to its west lies molecular cloud No. 7 of Yonekura et al. (2005). 


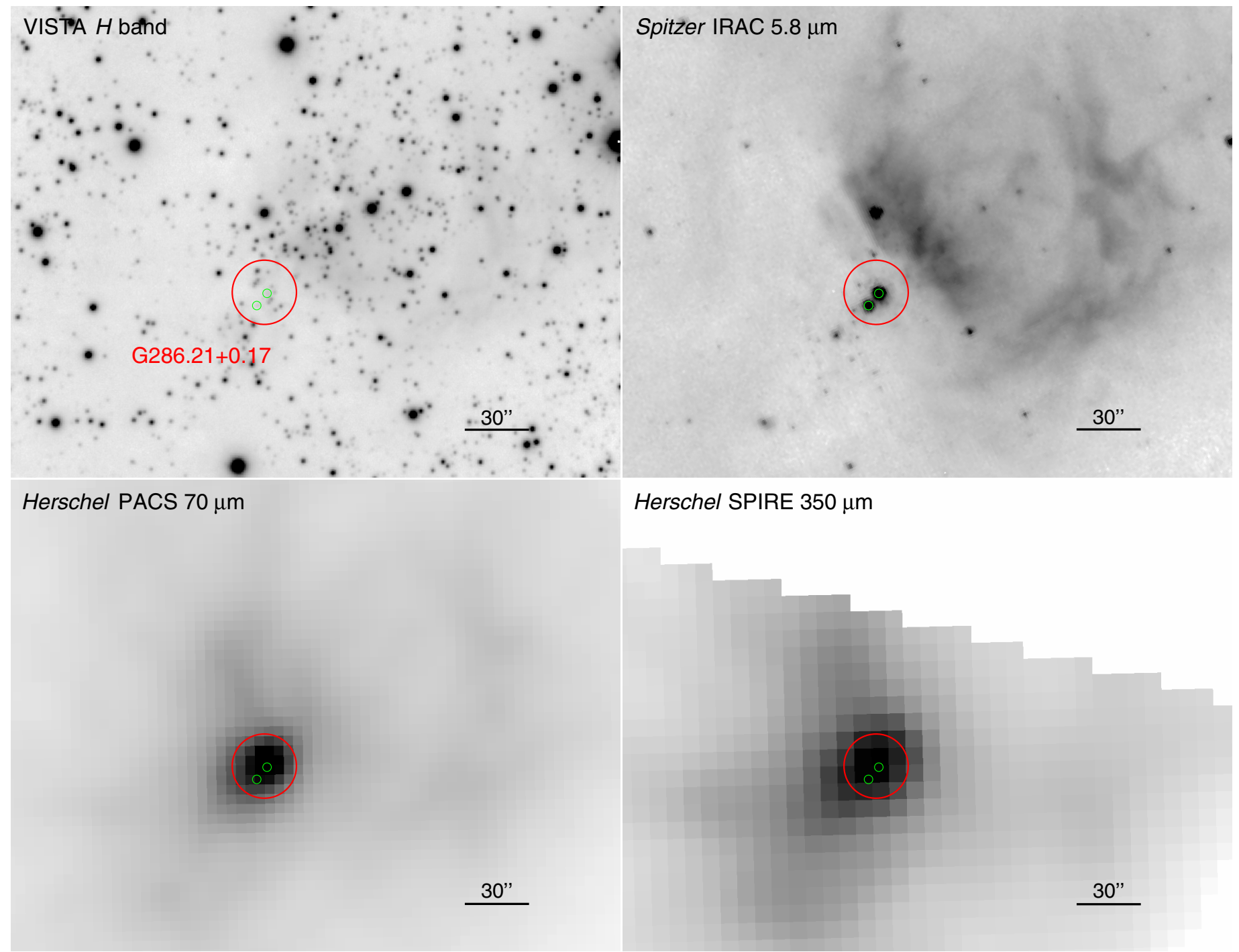

Fig. 11. Cluster G286.21+0.17 (red circle; cf. Sect. 4.4.4) and its immediate surroundings from near- to far-infrared. The two green circles mark the two bright Spitzer-resolved sources within it. (A close-up of the VISTA $H$-band image is shown in Fig. 10.)

It is accompanied by emission visible in the Herschel bands and a peculiarly green point-like feature in the IRAC RGB image, that is, strong emission in the IRAC $4.5 \mu \mathrm{m}$ band.

In the distribution of IRAC-identified cYSOs there is another conspicuous cluster consisting of 12 candidates to the west of the HII region around $\alpha_{2000}=10: 34: 27, \delta_{2000}=-58: 46: 45$ (orange circle in Fig. 8). This region is devoid of mid-infrared emission but is coincident with $\mathrm{C}^{18} \mathrm{O}$ clump No. 1 of Yonekura et al. (2005) and far-infrared emission as traced by Herschel. The cYSOs are aligned along the western ridge of the far-infrared cloud as seen in the Herschel image and even follow the shape of its filaments, broadly in the shape of an arrowhead pointing eastwards. The northern part is better aligned with the filament shape while the southern part is more randomly distributed around the filament itself. The border is also traced by the Yonekura et al. (2005) ${ }^{12} \mathrm{CO}$ intensity contours. According to WISE classification, however, there is nothing remarkable about that region. We find Class I candidate J103423.7-584531 to the north and Class II candidate J103424.6-584749 to the south, but no appearance of clustering.

North of the Gum 31 bubble, around $\alpha_{2000}=10: 37: 36$, $\delta_{2000}=-58: 26: 36$, there is a cluster of stars clearly discernible in the IRAC image (orange circle in Fig. 8). It lies about $2^{\prime}$ to the south-west of $\mathrm{C}^{18} \mathrm{O}$ clump No. 4 (Yonekura et al. 2005). Around thirty-five stars are seen within this group in projection and it is also associated with a number of Herschelidentified point-like sources. Two of them, J103739.6-582756 and J103741.7-582629, are part of the sample analysed in Sect. 5. Few stars within or around this cluster are identified as cYSOs with WISE. J103736.3-582655 and J103741.9-582556 are the brightest stars in the IRAC images of the cluster and are both identified as Class I candidates with WISE. There is one more Class I candidate and five Class II candidates distributed fairly evenly across the cluster.

There are several more minor clusters of $\sim 5$ cYSOs, notably always coincident with local maxima in the Yonekura et al. (2005) $\mathrm{C}^{18} \mathrm{O}$ maps.

\section{SED modelling for sources with both Herschel and Spitzer counterparts}

Using our Herschel and Spitzer catalogues, we were able to identify the sources that are detected as point-like sources in both wavelength ranges. For Herschel we applied the restriction that the sources had to be detected in at least three of the five bands, bringing the total number down from 91 sources detected in at least two bands to 59. This results in 17 sources overall 


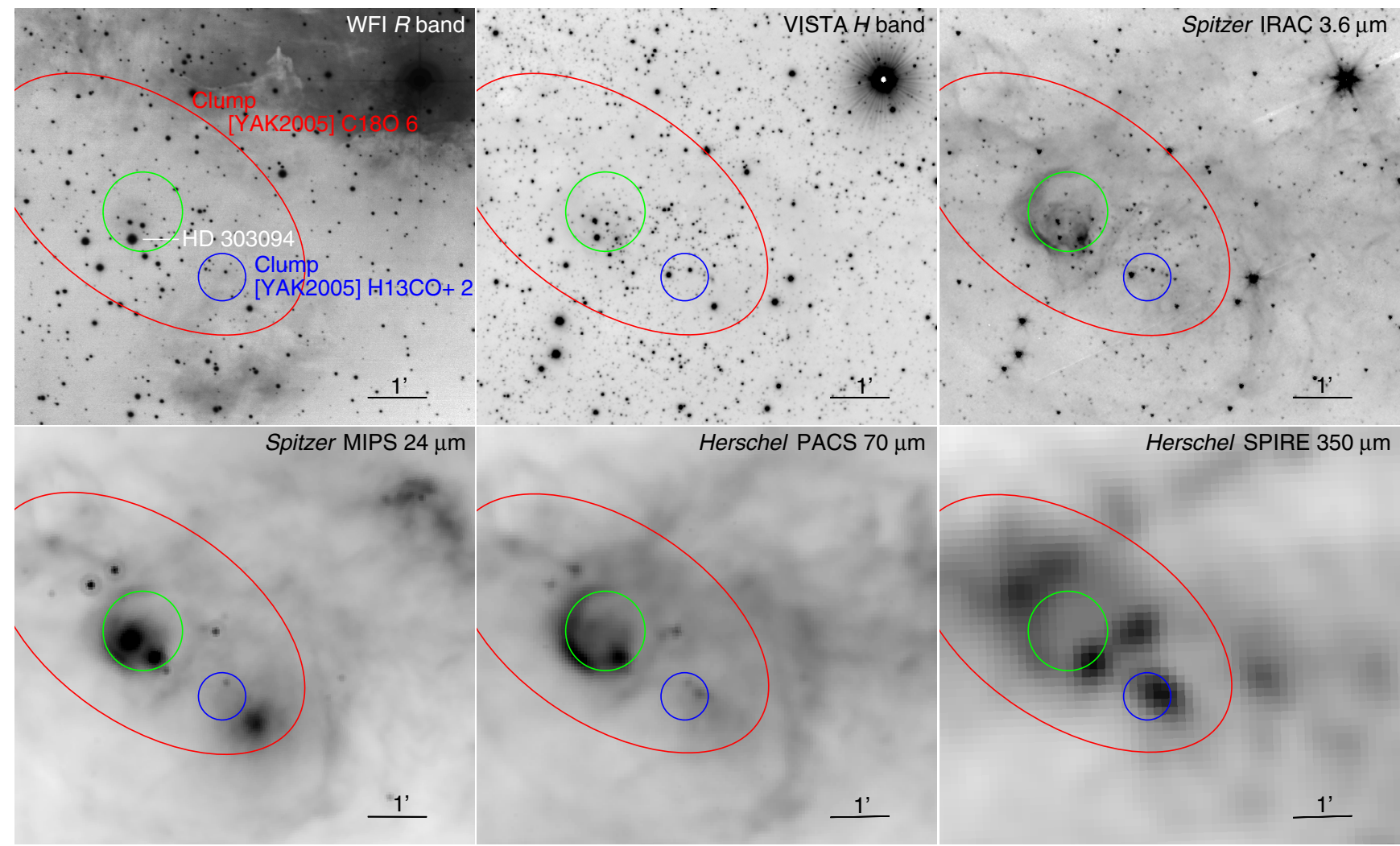

Fig. 12. Cluster G286.38-0.26 from near- to far-infrared. The large red ellipse marks the approximate outline of the C ${ }^{18} \mathrm{O}$ clump No. 6 ([YAK2005] C18O 6; Yonekura et al. 2005). The blue circle marks $\mathrm{H}^{13} \mathrm{CO}^{+}$clump No. 2 ([YAK2005] $\mathrm{H} 13 \mathrm{CO}+2$ ), the green circle indicates the shape of the arc-like nebulosity visible in the Spitzer IRAC images.

that can be identified in at least three Herschel bands and at least one of the IRAC bands. We then compared these identifications to the MIPS image and performed photometry as described in Sect. 2.1.2 for the nine sources where we could identify a MIPS counterpart.

To extend the wavelength range of our observations, we additionally matched the point sources analysed here with sources from the 2MASS All-Sky Catalog of Point Sources (Cutri et al. 2003). This was performed by applying the same search for the best match in position as for the inter-band matching within the IRAC sources (Sect. 2.1.1), using only sources with quality flags A to D. We then repeated this process with the WISE catalogue, where we selected only those sources that had a signal-tonoise ratio higher than 5. A detailed overview of all photometric data assembled is given in Table 2 in the online material. WISE $22 \mu \mathrm{m}$ and MIPS $24 \mu \mathrm{m}$ fluxes show some incongruity, but there is no underlying pattern as to what in the environs of the source may have influenced the photometry. This does not, however, influence the findings from the SED fits. In the two cases where the $22 \mu \mathrm{m}$ flux appears to be unduly high compared to the $24 \mu \mathrm{m}$ flux and an SED fit is performed, excluding one or the other from the fit has little or no influence on the best-fit model.

\subsection{Modelling of the SEDs}

For the SED-fitting we used the online tool of Robitaille et al. $(2007)^{8}$. This tool compares the input observational data with 200000 SED models for YSOs that were precomputed using a $2 \mathrm{D}$ radiative transfer code developed by Whitney et al. (2003).

\footnotetext{
8 http://caravan.astro.wisc.edu/protostars/sedfitter. php
}

These models have a wide parameter space for the properties of the central object and its environment.

For the fits, the distance to all objects was assumed to be $2.3 \mathrm{kpc}$ (cf. Sect. 3), and the interstellar extinction range was restricted to $A_{V}=0 \ldots 40 \mathrm{mag}$. We assumed an uncertainty of $20 \%$ for all Herschel fluxes. In addition to the individual photometric measurement uncertainty as given in Table 2, we assumed an additional systematic uncertainty of $10 \%$ for 2 MASS, Spitzer, and WISE fluxes due e.g. to the reliability of flux calibration. For IRAC, photometry varies by up to $10 \%$ due to the position of the point-like source within the detector array, and although appropriate corrections were applied in the process, this is an additional source of uncertainty.

In addition to the best-fit model, we show the range of possible parameters that can be derived from models within the range of $\chi^{2} / N-\chi_{\text {best }}^{2} / N<2$ (with $N$ representing the number of data points). These models are shown as grey lines in the plots in Fig. 13. The resulting model parameters are listed in Table 3 in the online material. It gives the best-fit value together with the range constrained by the above $\chi^{2}$ criterion. The resulting SEDs are shown in Fig. 13. We only used fits where $\chi^{2} / N$ for the bestfit model is smaller than or equal to 10.0.

\subsection{Results of SED modelling}

The results of SED fits can be highly ambiguous. Many of the stellar and circumstellar parameters are often poorly constrained because the models show a high degree of degeneracy (e.g. Men'shchikov \& Henning 1997). We therefore restricted our analysis to a few selected parameters that can be determined relatively accurately from these fits. These are the total luminosity, the stellar mass, and the mass of the circumstellar disk and envelope. 
H. Ohlendorf et al.: Discovering young stars in the Gum 31 region with infrared observations
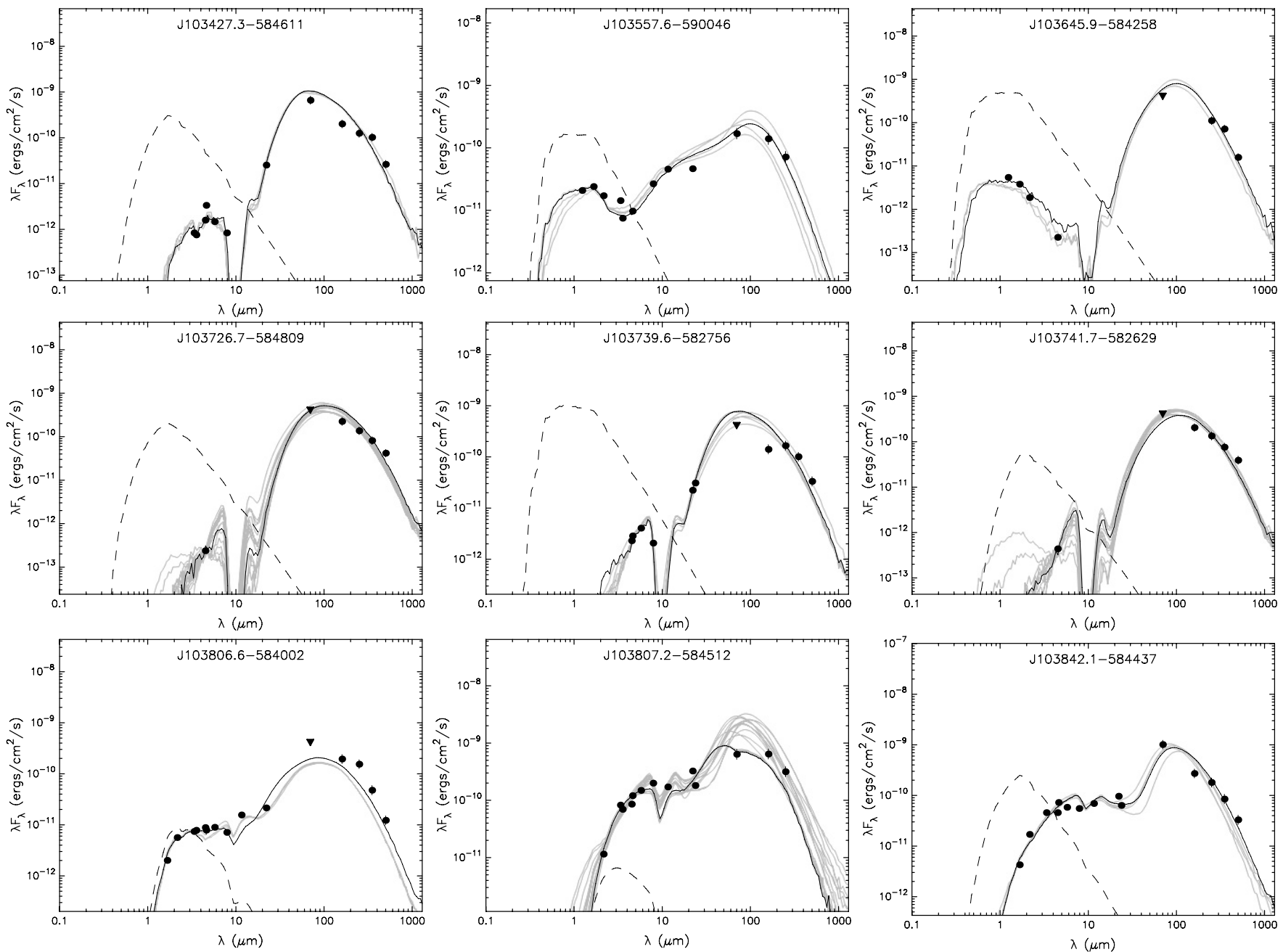

Fig. 13. Spectral energy distributions of the objects for which we could determine fluxes with at least three Herschel bands and one Spitzer IRAC band. Filled circles mark the input fluxes. The black line shows the best fit, the grey lines show subsequent good fits. The dashed line represents the stellar photosphere corresponding to the central source of the best-fitting model, as it would appear without circumstellar dust (but including interstellar extinction).

The best-fit masses as listed in Table 3 (online material) are between $1.7 M_{\odot}$ and $6.6 M_{\odot}$ and even the extremes of the ranges do not exceed $1.2 M_{\odot}$ to $7.3 M_{\odot}$. The majority of luminosities are to be found in a range of $\sim 100-300 L_{\odot}$, with two notable exceptions well below that at $38 L_{\odot}$ and $42 L_{\odot}$, and one exceptionally luminous source at $890 L_{\odot}$ best-fit value, corresponding to the highest best-fit stellar mass in our sample. While four of the ten sources sampled here exhibit best-fit envelope masses of $190 M_{\odot}$ or higher, three are at $\leq 100 M_{\odot}$ and two lower than $50 M_{\odot}$. The disk masses span a range of about one order of magnitude between $\sim 0.01 M_{\odot}$ and $\sim 0.1 M_{\odot}$. The highest-mass star in the sample is the notable exeption with a disk mass of $\sim 0.001 M_{\odot}$.

In a large-scale view it is immediately obvious that all but two of the sources for which Herschel counterparts to Spitzer point-like sources are detected are to be found within the Gum 31 bubble, although the field of our study stretches farther out especially to the west. These two sources outside the bubble are J103557.6-590046 and J103427.3-584611.

$\mathrm{J} 103806.6-584002$ is remarkable in that in contrast to the vast majority of objects it is not located in the rim of the bubble but within the bubble itself, the only such specimen in our sample. It is placed in the very head of a pillar-like filament that extends from the southern rim of the bubble into it (cf. Fig. 9). With the methods employed in Sect. 4 we classify it as a WISE Class I candidate and an IRAC cYSO. In the Herschel images the filament is quite faint, but J103806.6-584002 itself is clearly visible as a point-like source.

\section{Sources of $\mathrm{HH}$ jets}

In a previous paper (Ohlendorf et al. 2012) we have traced back a number of Herbig-Haro jets identified in the CNC by Smith et al. (2010a) to their protostellar sources. Smith et al. (2010a) also identified two $\mathrm{HH}$ jets and two $\mathrm{HH}$ jet candidates in the Gum 31 bubble. The HST images on which the study of Smith et al. is based cover only a very small area of the entire Gum 31 region, and the following analysis only represents a very small section of the area covered in the rest of this paper. Because of the small sample size and its very limited spatial dimensions, we cannot draw any conclusions about the distribution or likely number of all jet-driving protostars within the Gum 31 region.

As can be seen in Fig. 14, the jet HH 1002 is almost perpendicular to the ionisation front. It shows several distinct features, marked by Smith et al. (2010a) as A, B, and C and labelled thus in our figure. Smith et al. detected a very likely source located at $\alpha_{2000}=10: 36: 53.9, \delta_{2000}=-58: 37: 19$ in the 2MASS images. This almost exactly matches the location of the object identified in IRAC images that is most likely to be the source of the jet: J103654.0-583720, which we classified as being a cYSO by its WISE and IRAC infrared excesses in Sect. 4.1. The Herschel 

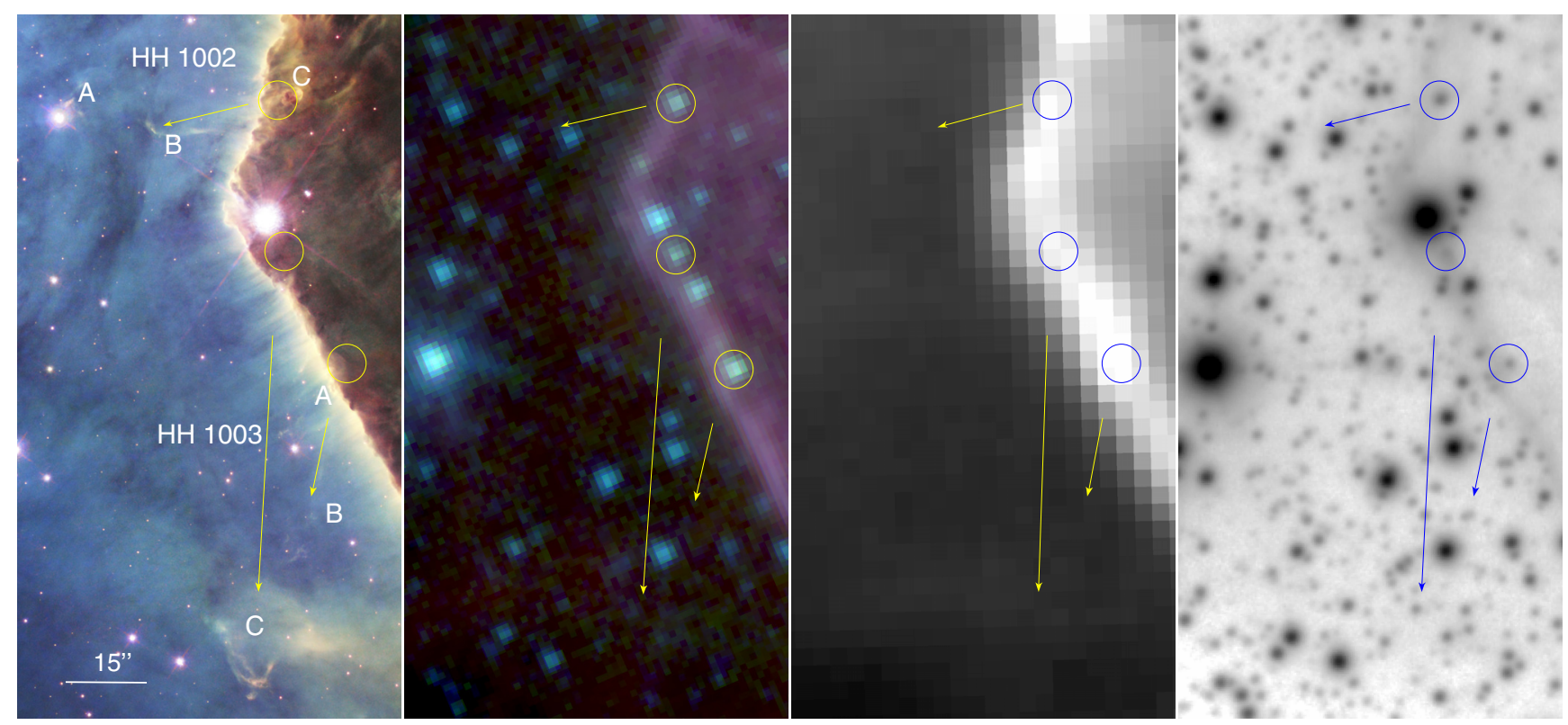

Fig. 14. Herbig-Haro jets and their probable sources as seen in four different wavelengths. From left to right: Hubble Space Telescope RGB image (red: WFPC2 S II filter $(673 \mathrm{~nm})$, green: ACS H $\alpha+\mathrm{N}$ II filter $(658 \mathrm{~nm})$, blue: WFPC2 O III filter $(502 \mathrm{~nm})$; image credit: NASA/ESA/Hubble Heritage Team (STScI/AURA)), Spitzer IRAC RGB image with $3.6 \mu \mathrm{m}$ in blue, $4.5 \mu \mathrm{m}$ in green, and $8.0 \mu \mathrm{m}$ in red, Herschel PACS $170 \mu \mathrm{m}$ image, and VISTA $H$-band image. The yellow or blue arrows indicate the broad shape and direction of the outflow in question, white letters mark their features as described by Smith et al. (2010a). The candidate infrared-identified sources are marked at their Spitzer coordinates with circles in yellow or blue.

observations trace the ionisation front well, but show no pointlike source coincident with J103654.0-583720. Therefore we did not include it in the SED-based analysis in Sect. 5.

HH 1003 has a more complicated structure; Smith et al. (2010a) suggested it might be a two-part object, consisting of two closely associated jets. Tracing back the direction of the bow shocks in Fig. 14, we find two point-like sources in the IRAC images that are very probably the sources of two different jets. One of them, J103652.4-583809, associated with features A and B, is identified as a cYSO from the WISE and IRAC colour-colour diagrams, while the other is an IRAC cYSO. Both are coincident with faint sources in the 2MASS images. Again, the Herschel image shows no point-like sources.

We were unable to trace back the two candidate outflows, HH c-1 and HHc-2 (not to be confused with HH c-1 and HHc-2 within the extent of the central Carina Nebula that were included in the study of Ohlendorf et al. 2012), to any infrared sources within the scope of our study. Following the axes of the jets, we cannot identify any likely emitting sources within their immediate surroundings.

\section{Discussion and conclusions}

We analysed Spitzer, WISE, and Herschel data to investigate the cloud structure and the young stellar population in and around the Gum 31 nebula. These data provide considerably better sensitivity and spatial resolution than previously available data sets.

The Herschel far-infrared maps show that the bubble surrounding the Gum 31 nebula is connected to the central parts of the Carina Nebula. This adds strong direct support to the assumption that Gum 31 is actually part of the CNC, as is also suggested by the matching $\mathrm{C}^{18} \mathrm{O}$ radial velocities measured by Yonekura et al. (2005) and other recent distance determinations (cf. Sect. 1).

The bubble itself has a very sharp western edge, at which the column density derived from Herschel measurements rises abruptly by at least an order of magnitude. The dust temperatures range from $\lesssim 20 \mathrm{~K}$ in the dark clouds surrounding the $\mathrm{H}$ II region to $\approx 30 \mathrm{~K}$ in the $\mathrm{HII}$ region and up to $\approx 40 \mathrm{~K}$ near the location of the O-type stars.

The very small excess fraction seen for the mid-infrared sources in the central stellar cluster NGC $3324(\approx 0.5 \%)$ suggests that it is at least several Myr old. For the whole Gum 31 region, the Spitzer and WISE data reveal about 300/660 cYSOs. These objects are most likely Class I protostars or Class II sources of solar mass to intermediate mass. The 59 far-infrared point-like sources revealed by the Herschel data are either pre-stellar cores or embedded (Class 0) protostars, i.e. trace a younger population of currently forming stars. The spatial distribution of the cYSOs is highly non-uniform. We expect a contamination of around $18 \%$, so most of the widely scattered YSO population we see is probably due to back- and foreground stars which would be expected to be distributed homogeneously. Many cYSOs are found in rather compact clusterings, and a considerable number is found at the inner edge of the dusty bubble surrounding the $\mathrm{H}$ II region. The Herschel-identified point-like sources in particular trace the edge of the bubble.

This led us to assume a collect-and-collapse scenario driven by the O stars within the cluster (Whitworth et al. 1994), resulting in an expected bubble size of $9-11 \mathrm{pc}$ for an age of 1.5-2.0 Myr. This agrees very well with the observed diameter of $\sim 10 \mathrm{pc}$. We find four cYSOs in the very tips of short pillars extending from the bubble rim (cf. Fig. 9), suggesting radiative triggering processes very similar to what is observed in the South Pillars (Smith et al. 2010b; Gaczkowski et al. 2013).

We concluded that two different modes of triggered starformation occur simultaneously in the Gum 31 region: collectand-collapse, as shown by the bubble size and the cYSOs in its rim, and radiative triggering, as shown by cYSOs in the heads of pillars.

We constructed the near- to far-infrared SEDs of 17 cYSOs and estimated basic stellar and circumstellar parameters by comparison to radiative-transfer models with good-quality fits 
for nine of them. All these cYSOs are of moderate luminosity $\left(L \lesssim 900 L_{\odot}\right)$, clearly suggesting that they are low- or intermediate-mass objects $\left(M \lesssim 7 M_{\odot}\right)$. This agrees with the results from our analysis of the cYSOs in the central parts of the CNC (Gaczkowski et al. 2013), where we found that no highmass stars are currently forming.

We identified the driving sources of two Herbig-Haro jets in the western rim of the Gum 31 bubble. These sources were also identified as cYSOs by applying colour-selection criteria to IRAC or WISE photometry data.

From the total number of cYSOs observed and the IMF (Kroupa 2002) we can estimate a total young stellar population for the Gum 31 region. Our detection limit for cYSOs is about $1 M_{\odot}$ and following the IMF there should be eight times as many stars below this mass $\left(>0.1 M_{\odot}\right)$ as above it. Correcting the number of cYSOs given in Sect. 4.2 for the contamination estimated there, this yields $\sim 5000$ young stellar objects in the region.

A more detailed investigation of the star formation history in this area requires a reliable identification of the individual young stars. While the infrared data presented in this paper can reveal protostars and young stars with circumstellar disks, most of the slightly older ( $~ 22$ Myr old) stars cannot be detected by infrared excesses. Our very recent Chandra X-ray observations of the Gum 31 region, near-infrared observations with VISTA, and other ongoing observations will allow us to finally identify these stars, too. This will constitute the basis for a comprehensive multi-wavelength study of this interesting region, in a way similar to the recent studies of the young stellar populations in the central parts of the Carina Nebula (see Townsley et al. 2011; Preibisch et al. 2011a; Wang et al. 2011; Wolk et al. 2011; Feigelson et al. 2011).

Acknowledgements. This work was supported by the German Deutsche For schungsgemeinschaft, DFG project number 569/9-1. Additional support came from funds from the Munich Cluster of Excellence "Origin and Structure of the Universe". The authors would like to thank the Spitzer Science Center Helpdesk for support while working with MOPEX. This work is based in part on archival data obtained with the Spitzer Space Telescope, which is operated by the Jet Propulsion Laboratory, California Institute of Technology under a contract with NASA. This publication makes use of data obtained with the Herschel spacecraft. The Herschel spacecraft was designed, built, tested, and launched under a contract to ESA managed by the Herschel/Planck Project team by an industrial consortium under the overall responsibility of the prime contractor Thales Alenia Space (Cannes), and including Astrium (Friedrichshafen) responsible for the payload module and for system testing at spacecraft level, Thales Alenia Space (Turin) responsible for the service module, and Astrium (Toulouse) responsible for the telescope, with in excess of a hundred subcontractors. This publication makes use of data products from the Two Micron All Sky Survey, which is a joint project of the University of Massachusetts and the Infrared Processing and Analysis Center/California Institute of Technology, funded by the National Aeronautics and Space Administration and the National Science Foundation. This publication makes use of data products from the Wide-field Infrared Survey Explorer, which is a joint project of the University of California, Los Angeles, and the Jet Propulsion Laboratory/California Institute of Technology, funded by the National Aeronautics and Space Administration. The science data reduction for VISTA up to the creation of the final tile was performed by the Cambridge Astronomy Survey Unit (see http://casu.ast.cam.ac.uk/ surveys-projects/vista/data-processing for details). This research has made use of NASA's Astrophysics Data System Bibliographic Services. This research has made use of the SIMBAD database and the VizieR catalogue access tool, operated at CDS, Strasbourg, France.

\section{References}

Allen, L. E., Calvet, N., D'Alessio, P., et al. 2004, ApJS, 154, 363 Baraffe, I., Chabrier, G., Allard, F., \& Hauschildt, P. H. 1998, A\&A, 337, 403 Barnes, P. J., Yonekura, Y., Ryder, S. D., et al. 2010, MNRAS, 402, 73 Baumgardt, H., Dettbarn, C., \& Wielen, R. 2000, A\&AS, 146, 251
Cappa, C., Niemela, V. S., Amorín, R., \& Vasquez, J. 2008, A\&A, 477, 173 Carraro, G., Patat, F., \& Baumgardt, H. 2001, A\&A, 371, 107

Clariá, J. J. 1977, A\&AS, 27, 145

Cutri, R. M., et al. 2012, VizieR Online Data Catalog: II/311

Cutri, R. M., Skrutskie, M. F., van Dyk, S., et al. 2003, VizieR Online Data Catalog: II/246

Cutri, R. M., Wright, E. L., Conrow, T., et al. 2012, Explanatory Supplement to the WISE All-Sky Data Release Products, Tech. rep.

Dalton, G. B., Caldwell, M., Ward, A. K., et al. 2006, in SPIE Conf. Ser., 6269 Dewangan, L. K., Ojha, D. K., Anandarao, B. G., Ghosh, S. K., \& Chakraborti, S. 2012, ApJ, 756, 151

Diolaiti, E., Bendinelli, O., Bonaccini, D., et al. 2000, A\&AS, 147, 335

Dullemond, C. P., \& Dominik, C. 2004, A\&A, 417, 159

Dutra, C. M., Bica, E., Soares, J., \& Barbuy, B. 2003, A\&A, 400, 533

Emerson, J., \& Sutherland, W. 2010, The Messenger, 139, 2

Fang, M., van Boekel, R., King, R. R., et al. 2012, A\&A, 539, A119

Faúndez, S., Bronfman, L., Garay, G., et al. 2004, A\&A, 426, 97

Fazio, G. G., Hora, J. L., Allen, L. E., et al. 2004, ApJS, 154, 10

Feigelson, E. D., Getman, K. V., Townsley, L. K., et al. 2011, ApJS, 194, 9

Flaherty, K. M., Pipher, J. L., Megeath, S. T., et al. 2007, ApJ, 663, 1069

Forte, J. C. 1976, A\&AS, 25, 271

Gaczkowski, B., Preibisch, T., Ratzka, T., et al. 2013, A\&A, 549, A67

Griffin, M. J., Abergel, A., Abreu, A., et al. 2010, A\&A, 518, L3

Hoffleit, D. 1953, Annals of Harvard College Observatory, 119, 37

Irwin, M. J., Lewis, J., Hodgkin, S., et al. 2004, in SPIE Conf. Ser., eds. P. J. Quinn, \& A. Bridger, 5493, 411

Kharchenko, N. V., Piskunov, A. E., Röser, S., Schilbach, E., \& Scholz, R.-D 2005, A\&A, 438, 1163

Koenig, X. P., Leisawitz, D. T., Benford, D. J., et al. 2012, ApJ, 744, 130

Kramer, C., Cubick, M., Röllig, M., et al. 2008, A\&A, 477, 547

Kroupa, P. 2002, Science, 295, 82

Kudritzki, R. P., Puls, J., Lennon, D. J., et al. 1999, A\&A, 350, 970

Lada, C. J. 1987, in Star Forming Regions, ed. M. Peimbert, \& J. Jugaku, IAU Symp., 115,1

Lyngå, G., \& Hansson, N. 1972, A\&AS, 6, 327

Maíz-Apellániz, J., Walborn, N. R., Galué, H. Á., \& Wei, L. H. 2004, ApJS, 151, 103

Makovoz, D., \& Marleau, F. R. 2005, PASP, 117, 1113

Men'shchikov, A. B., \& Henning, T. 1997, A\&A, 318, 879

Molinari, S., Schisano, E., Faustini, F., et al. 2011, A\&A, 530, A133

Mottram, J. C., Hoare, M. G., Lumsden, S. L., et al. 2007, A\&A, 476, 1019

Nesterov, V. V., Kuzmin, A. V., Ashimbaeva, N. T., et al. 1995, A\&AS, 110, 367

Ohlendorf, H., Preibisch, T., Gaczkowski, B., et al. 2012, A\&A, 540, A81

Oliveira, I., Merín, B., Pontoppidan, K. M., et al. 2009, ApJ, 691, 672

Pickles, A., \& Depagne, É. 2010, PASP, 122, 1437

Poglitsch, A., Waelkens, C., Geis, N., et al. 2010, A\&A, 518, L2

Preibisch, T., Hodgkin, S., Irwin, M., et al. 2011a, ApJS, 194, 10

Preibisch, T., Ratzka, T., Kuderna, B., et al. 2011b, A\&A, 530, A34

Preibisch, T., Schuller, F., Ohlendorf, H., et al. 2011c, A\&A, 525, A92

Preibisch, T., Roccatagliata, V., Gaczkowski, B., \& Ratzka, T. 2012, A\&A, 541, A132

Przybilla, N., Butler, K., Becker, S. R., \& Kudritzki, R. P. 2006, A\&A, 445, 1099

Ragan, S., Henning, T., Krause, O., et al. 2012, A\&A, 547, A49

Rieke, G. H., Young, E. T., Engelbracht, C. W., et al. 2004, ApJS, 154, 25

Robitaille, T. P., Whitney, B. A., Indebetouw, R., \& Wood, K. 2007, ApJS, 169 328

Salatino, M., de Bernardis, P., Masi, S., \& Polenta, G. 2012, ApJ, 748, 1

Skrutskie, M. F., Cutri, R. M., Stiening, R., et al. 2006, AJ, 131, 1163

Smith, N. 2006a, MNRAS, 367, 763

Smith, N. 2006b, MNRAS, 368, 1983

Smith, N. 2006c, ApJ, 644, 1151

Smith, N., \& Brooks, K. J. 2007, MNRAS, 379, 1279

Smith, N., \& Brooks, K. J. 2008, Handbook of Star Forming Regions, Volume II, ed. B. Reipurth, 138

Smith, N., Bally, J., \& Walborn, N. R. 2010a, MNRAS, 405, 1153

Smith, N., Povich, M. S., Whitney, B. A., et al. 2010b, MNRAS, 406, 952

Townsley, L. K., Broos, P. S., Corcoran, M. F., et al. 2011, ApJS, 194, 1

Wang, J., Feigelson, E. D., Townsley, L. K., et al. 2011, ApJS, 194, 11

Whitney, B. A., Wood, K., Bjorkman, J. E., \& Wolff, M. J. 2003, ApJ, 591, 1049

Whitworth, A. P., Bhattal, A. S., Chapman, S. J., Disney, M. J., \& Turner, J. A. 1994, A\&A, 290, 421

Winston, E., Megeath, S. T., Wolk, S. J., et al. 2007, ApJ, 669, 493

Wolk, S. J., Broos, P. S., Getman, K. V., et al. 2011, ApJS, 194, 12

Wright, E. L., Eisenhardt, P. R. M., Mainzer, A. K., et al. 2010, AJ, 140, 1868

Yonekura, Y., Asayama, S., Kimura, K., et al. 2005, ApJ, 634, 476

Pages 18 to 20 are available in the electronic edition of the journal at http: //www . aanda.org 
Table 1. Overview of observations with Spitzer and Herschel.

\begin{tabular}{lllcll}
\hline \hline Instrument & AOR & Observation mode & Observation time & Date & PI \\
\hline Spitzer IRAC & 23708160 & IRAC map & $3301 \mathrm{~s}$ & 19 July 2008 & S. R. Majewski \\
Spitzer IRAC & 23699200 & IRAC map & $3057 \mathrm{~s}$ & 19 July 2008 & S. R. Majewski \\
Spitzer IRAC & 23704320 & IRAC map & $3070 \mathrm{~s}$ & 19 July 2008 & S. R. Majewski \\
Spitzer IRAC & 23688192 & IRAC map & $3082 \mathrm{~s}$ & 19 July 2008 & S. R. Majewski \\
Spitzer IRAC & 23701504 & IRAC map & $3086 \mathrm{~s}$ & 19 July 2008 & S. R. Majewski \\
Spitzer IRAC & 23695360 & IRAC map & $3086 \mathrm{~s}$ & 19 July 2008 & S. R. Majewski \\
Spitzer IRAC & 23696896 & IRAC map & $3087 \mathrm{~s}$ & 19 July 2008 & S. R. Majewski \\
Spitzer IRAC & 23706368 & IRAC map & $3088 \mathrm{~s}$ & 19 July 2008 & S. R. Majewski \\
Spitzer IRAC & 23684352 & IRAC map & $3312 \mathrm{~s}$ & 19 July 2008 & S. R. Majewski \\
Spitzer MIPS & 15054080 & MIPS phot & $9469 \mathrm{~s}$ & 12 June 2006 & J. Hester \\
Herschel PACS & 1342211615 & SpirePacsParallel & $11889 \mathrm{~s}$ & 26 December 2010 & T. Preibisch \\
Herschel PACS & 1342211616 & SpirePacsParallel & $12863 \mathrm{~s}$ & 26 December 2010 & T. Preibisch \\
Herschel SPIRE & 1342211615 & SpirePacsParallel & $11889 \mathrm{~s}$ & 26 December 2010 & T. Preibisch \\
Herschel SPIRE & 1342211616 & SpirePacsParallel & $12863 \mathrm{~s}$ & 26 December 2010 & T. Preibisch \\
\hline
\end{tabular}


H. Ohlendorf et al.: Discovering young stars in the Gum 31 region with infrared observations

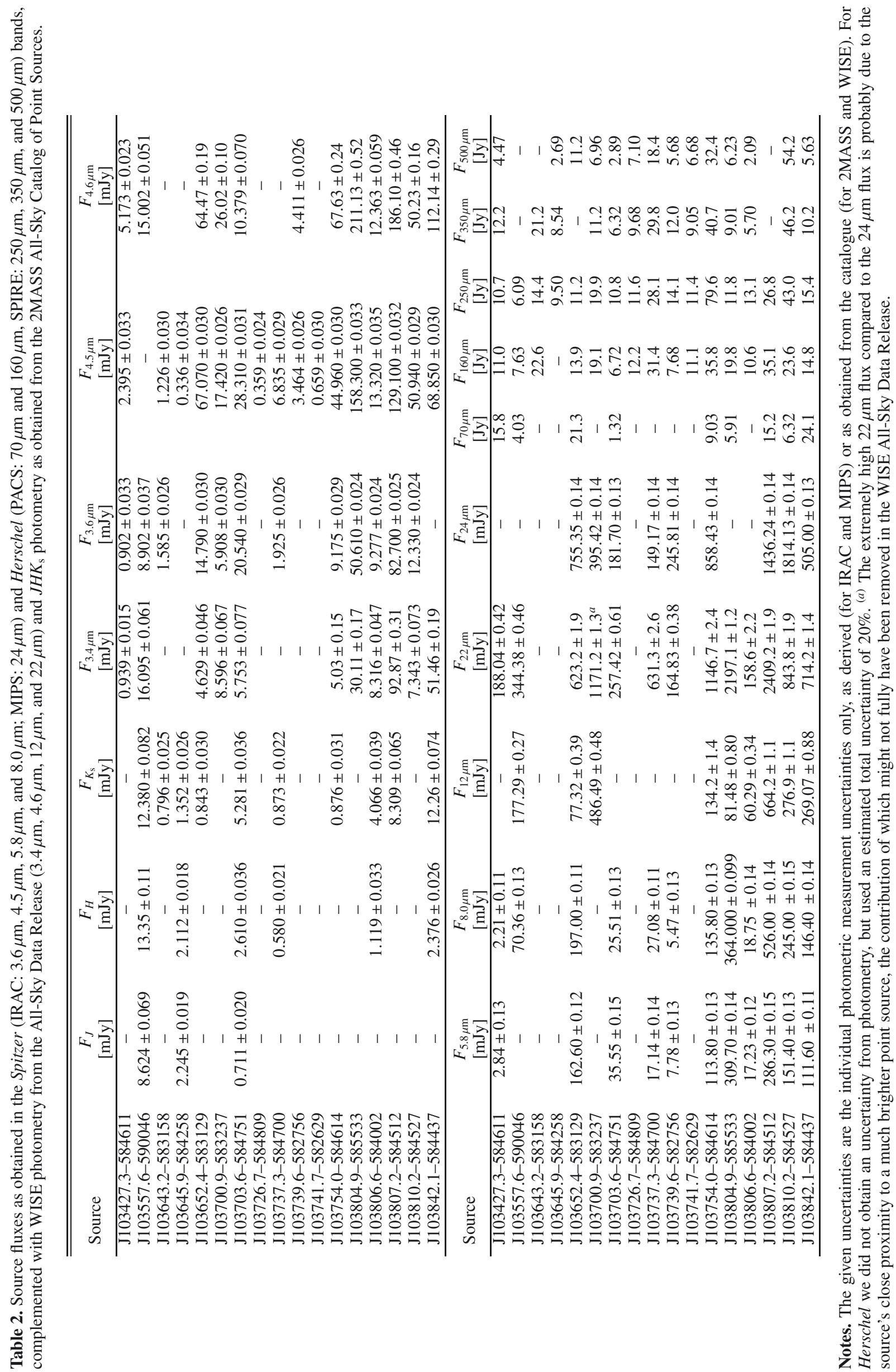




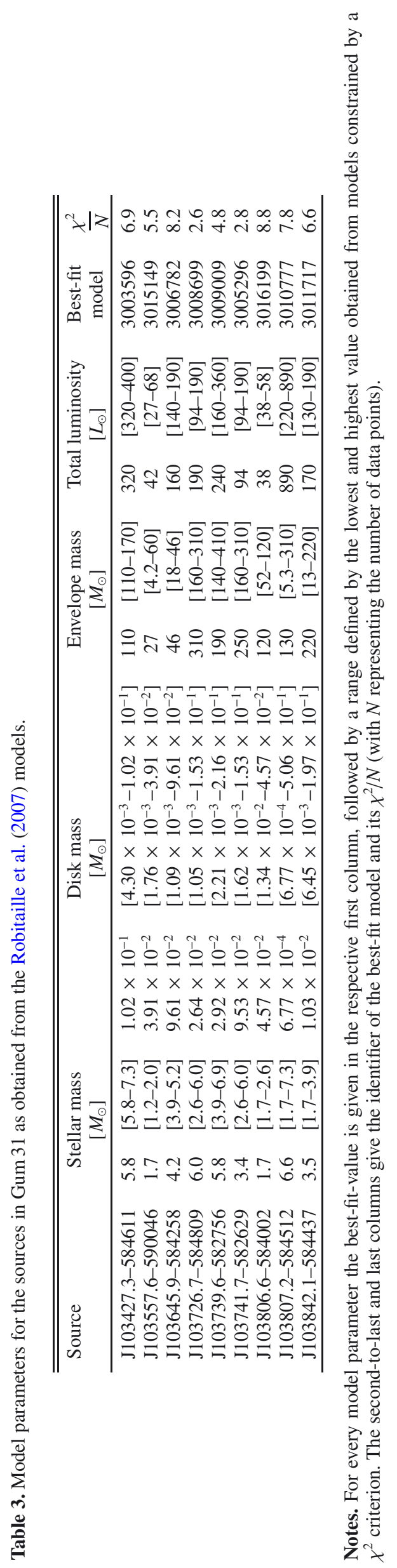

